\title{
Betti multiplets, flows across dimensions and $c$-extremization
}

\author{
Antonio Amariti ${ }^{a, b}$ and Chiara Toldo ${ }^{c}$ \\ ${ }^{a}$ Physics Department, The City College of the CUNY, \\ 160 Convent Avenue, New York, NY 10031, U.S.A. \\ ${ }^{b}$ Albert Einstein Center for Fundamental Physics, Institute for Theoretical Physics, \\ University of Bern, Sidlerstrasse 5, Bern, ch-3012, Switzerland \\ ${ }^{c}$ Columbia University in the City of New York, \\ 538 West 120th Street, 704 Pupin Hall, MC 5225, New York, NY 10027, U.S.A. \\ E-mail: amariti@itp.unibe.ch, ct2673@columbia.edu
}

AbSTRACT: We consider $4 \mathrm{~d} \mathcal{N}=1$ SCFTs, topologically twisted on compact constant curvature Riemann surfaces, giving rise to $2 \mathrm{~d} \mathcal{N}=(0,2)$ SCFTs. The exact R-current of these $2 \mathrm{~d}$ SCFT extremizes the central charge $c_{2 d}$, similarly to the $4 \mathrm{~d}$ picture, where the exact R-current maximizes the central charge $a_{4 d}$. There are global currents that do not mix with the R-current in $4 \mathrm{~d}$ but their mixing becomes non trivial in $2 \mathrm{~d}$. In this paper we study the holographic dual of this process by analyzing a $5 \mathrm{~d} \mathcal{N}=2$ truncation of $T^{1,1}$ with one Betti vector multiplet, dual to the baryonic current on the CFT side. The holographic realization of the flow across dimensions connects $\mathrm{AdS}_{5}$ to $\mathrm{AdS}_{3}$ vacua in the supergravity picture. We verify the existence of the flow to $\mathrm{AdS}_{3}$ solutions and we retrieve the field theory results for the mixing of the Betti vector with the graviphoton. Moreover, we extract the central charge from the Brown-Henneaux formula, matching with the results obtained in field theory. We develop a general formalism to obtain the central charge of a $2 \mathrm{~d}$ SCFT from $5 \mathrm{~d} \mathcal{N}=2$ gauged supergravity with a generic number of vector multiplets, showing that its extremization corresponds to an attractor mechanism for the scalars in the supergravity picture.

Keywords: AdS-CFT Correspondence, Conformal Field Theory, Field Theories in Lower Dimensions, Supergravity Models

ARXIV EPRINT: 1610.08858 


\section{Contents}

1 Introduction 1

2 Flowing from $4 \mathrm{~d} \mathcal{N}=1$ to $2 \mathrm{~d} \mathcal{N}=(0,2)$ SCFTs 3

3 Flowing from $\mathrm{AdS}_{5}$ to $\mathrm{AdS}_{3}$ in $\mathcal{N}=2$ gauged supergravity 6

3.1 Inclusion of the hypermultiplets 9

$4 \mathcal{N}=4$ SYM and $\mathrm{U}(1)^{3}$ gauged supergravity 10

$5 T^{1,1}$ with a Betti multiplet $r$

5.1 The $5 \mathrm{~d}$ truncation 11

$\begin{array}{ll}\text { 5.2 The ansatz and the BPS equations } & 13\end{array}$

$\begin{array}{lll}5.3 & \text { The } \mathrm{AdS}_{3} \text { vacuum } & 14\end{array}$

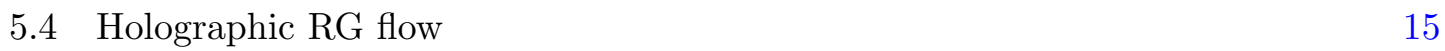

$\begin{array}{lll}5.5 & c \text {-extremization from gauged supergravity } & 17\end{array}$

$\begin{array}{llr}6 & \text { M5 branes } & 18\end{array}$

$\begin{array}{llr}7 \text { Conclusions } & 19\end{array}$

$\begin{array}{ll}\text { A } \mathcal{N}=2 \text { 5d gauged supergravity } & 21\end{array}$

B BPS equations $\quad 23$

B.1 Gravitino 23

B.2 Gaugino 24

B.3 Hyperino 24

\section{Introduction}

A useful mechanism to construct SCFTs in $d$-dimensions consists in compactifying a $(d+n)$ dimensional superconformal theory on a compact $n$-dimensional manifold $\mathcal{M}_{n}$. By turning on background magnetic fluxes for the global symmetries some amount of supersymmetry of the $d+n$ dimensional theory can be preserved in $d$ dimensions. This procedure has been developed in [1] and it is usually referred as (partial) topological twist.

The topological twists does not guarantee the existence of a superconformal symmetry in the lower dimensional theory. A powerful hint for the existence of such symmetry is provided by the AdS/CFT correspondence. Let us consider a four dimensional $\mathcal{N}=$ 1 SCFT, with an $\mathrm{AdS}_{5}$ gravity dual description, and compactify such model on a two dimensional compact Riemann surface of genus $\mathfrak{g}, \Sigma_{\mathfrak{g}}$. The existence of a $\mathcal{N}=(0,2)$ SCFT 
in two dimensions can be inferred from the existence of the gravitational flow from $\mathrm{AdS}_{5}$ to $\mathrm{AdS}_{3} \times \Sigma_{g}$. Finding an explicit gravitational flow usually requires the knowledge of the full 10 dimensional geometry. A rich variety of examples has been worked out in the literature, see for example [2-15]. For classifications of $\mathrm{AdS}_{3}$ solutions with uplift in 10d and $11 \mathrm{~d}$ see [16] and [17].

In particular in [12] the authors studied the gravity dual of an infinite set of quiver gauge theories, namely $Y^{p q}$ theories [18]. They arise by the worldvolume gauge theory living on a stack of $N$ D3 branes probing the tip of a three dimensional Calabi-Yau cone with a five dimensional Sasaki Einstein $\left(\mathrm{SE}_{5}\right)$ base, in this case a $Y^{p q}$ manifold [19]. Such theories are $\mathcal{N}=1$ quiver gauge theories in $4 \mathrm{~d}$ and, by turning on background magnetic fluxes for the global symmetries, it has been shown that in two dimensions they can flow to theories with $\mathcal{N}=(0,2)$ supersymmetry. The possible existence of a superconformal phase has been shown for suitable choices of the quantized fluxes. This has been checked by studying the central charge on the field theory side and matching with the solutions on the dual gravity side.

This analysis revealed an interesting feature regarding the exact R-current of the $2 \mathrm{~d}$ $\mathcal{N}=(0,2)$ theories. As in the $4 \mathrm{~d}$ case indeed the exact R-current is a linear combinations of the UV R-current and of the abelian global symmetries. In $4 \mathrm{~d}$ the mixing coefficients can be obtained by maximizing the conformal anomaly $a_{4 d}[20]$. In the $Y^{p q}$ case there the are in general two possible sources of mixing: the mesonic symmetries and the baryonic ones. A feature of these models is that the baryonic symmetry does not contribute to the mixing, ${ }^{1}$ namely the exact R-current in $4 \mathrm{~d}$ is a linear combination of the UV R-current and of the (mesonic) flavor symmetries only. However, after the compactification of the theories on $\Sigma_{g}$ the baryonic symmetry in general contributes to the exact R-current of the $2 \mathrm{~d}$ theory [12]. The mixing coefficient is computed by extremizing the two dimensional central charge.

This mechanism can be captured on the supergravity side, by considering a $5 \mathrm{~d}$ model arising from a consistent truncation of $10 \mathrm{~d}$ supergravity. The baryonic symmetry is associated to the presence of a so called Betti vector multiplet in the spectrum of the five dimensional $\mathcal{N}=2$ gauged supergravity. ${ }^{2}$ Hence by considering a truncation with a graviton and a Betti vector multiplet one should see the absence of mixing on the $5 \mathrm{~d}$ theory and the presence of such mixing after the flow to $3 \mathrm{~d}$.

In this paper we check this idea by studying an explicit truncation of the $Y^{10}=T^{1,1}$ theory, dual to the Klebanov Witten theory [24]. The supergravity truncation was provided in [25] (see also [26]) and it requires in particular the presence of hypermultiplets and massive vectors. We develop the necessary formalism in $5 \mathrm{~d} \mathcal{N}=2$ gauged supergravity to study the flow across dimensions in presence of background fluxes and obtain the $2 \mathrm{~d}$ central charge in terms of the sections, the isometries and the fluxes. Notice that the relation between $c$-extremization and the $\mathcal{N}=2$ gauged supergravity BPS flow equations has already been proposed in [27], where the $S T U$ truncation (one graviton and two massless

\footnotetext{
${ }^{1}$ See [21] for a discussion of the mixing of baryonic symmetries in the $Y^{21}$ model, and for example [22] for a general discussion on the mixing in $\mathrm{SE}_{5}$ manifolds.

${ }^{2}$ See also [23] for a recent analysis of the Betti multiplets in these models.
} 
vector multiplets) was taken into consideration. Here we consider a more general setup, without fixing the number of vector multiplets and discussing the analysis in presence of hypermultiplets.

The paper is organized as follows. In section 2 we review the compactification of $4 \mathrm{~d}$ $\mathcal{N}=1$ SCFTs on compact Riemann surfaces by a partial topological twist. We focus on the Klebanov Witten theory, reproducing the formula for $c_{2 d}$ in presence of a twist along the baryonic symmetry. In section 3 we study the flows across dimensions from the perspective of gauged supergravity in presence of a graviton and $n_{V}$ vector multiplets. We concentrate on the analysis of the central charges of the dual SCFTs from the gravitational perspective. We derive a general formula for $c_{2 d}$ in terms of the gravitational quantities and show that its extremization corresponds to an attractor mechanism in supergravity. We conclude the analysis by discussing how the formalism is modified by the presence of matter hypermultiplets in subsection 3.1. Sections 4, 5, 6 are devoted to the discussion of specific examples this formulation can be applied to. In section 4 we apply the formalism to the compactification of $\mathcal{N}=4 \mathrm{SYM}$ with a generic twist, breaking supersymmetry to $\mathcal{N}=(0,2)$ in $2 \mathrm{~d}$, and match our formula with the results of [8]. In section 5 we discuss the main result of our paper. We study a truncation of $T^{1,1}$ in presence of a Betti vector multiplet. For consistency the truncation requires also the presence of a massive vector multiplet and hypermultiplets. We study the compactification on the Riemann surface on the supergravity side, finding the holographic RG flow to the $\mathrm{AdS}_{3}$ vacuum. Then we compute $c_{2 d}$ from supergravity, with the techniques developed in section 3 , reproducing the field theory results and showing the mixing of the Betti multiplet with the graviphoton. In section 6 we apply our formalism to another example, corresponding to the truncation of $7 \mathrm{~d} \mathcal{N}=4 \mathrm{SO}(5)$ gauged supergravity reduced on a Riemann surface. This truncation is dual to the infinite family of $4 \mathrm{~d} \mathcal{N}=1$ SCFTs associated to M5 branes worked out in [28]. In this case we recover the large $N$ results already obtained in $[8,10]$. In section 7 we conclude and discuss possible lines of research. Two appendices clarify notations and details of the calculations. In appendix A we provide a review of gauged supergravity, fixing the conventions used in the paper. In appendix B we provide the details of derivation of the BPS equations for the truncation of $T^{1,1}$ with the Betti vector multiplet on a constant curvature Riemann surface.

Note added: when finishing this paper we noticed [29] on the ArXiv, where attractor equations for extremal black strings in presence of hypermultiplets have been derived via rewriting the action in a sum of squares, and a prescription to compute $c_{2 d}$ from $5 \mathrm{~d} \mathcal{N}=2$ gauged supergravity is provided.

\section{Flowing from $4 \mathrm{~d} \mathcal{N}=1$ to $2 \mathrm{~d} \mathcal{N}=(0,2)$ SCFTs}

In this section we discuss some aspects of the flow across dimensions discussed in [12]. More concretely we sketchily review the derivation of the central charge $c_{2 d}$ from $4 \mathrm{~d}$ SCFTs on two dimensional compact Riemann surfaces with a partial topological twist along the abelian symmetries. 
Consider a four dimensional $\mathcal{N}=1 \mathrm{SCFT}$. At the fixed point the exact $\mathrm{R}$-current is determined by a linear combination of the abelian flavor symmetries $J_{F_{i}}$ and of the UV R-current, $R_{0}$

$$
R=R_{0}+\alpha_{i} J_{F_{i}}
$$

The exact coefficients $\alpha_{i}$ of such mixing are determined by maximizing the central charge $a_{4 d}[20]$

$$
a_{4 d}=\frac{3}{32}\left(3 \operatorname{Tr} R^{3}-\operatorname{Tr} R\right) .
$$

Such a superconformal theory can be compactified on a genus $\mathfrak{g}$ compact Riemann surface $\Sigma_{\mathfrak{g}}$ : some supersymmetries can be preserved if some background magnetic fluxes are turned on, canceling the spin connection on the curved manifold. In general one can turn on such background fluxes for all the non anomalous global abelian symmetries of the $4 \mathrm{~d}$ theory. ${ }^{3}$

Define $T_{i}$ as the generator of the $i$-th abelian symmetry. One turns on the external fluxes as $F_{i}=T_{i} v l_{\Sigma_{\mathfrak{g}}}$, where $\operatorname{vol}_{\Sigma_{\mathfrak{g}}}$ is the volume form, normalized such that $\int d v o l_{\Sigma_{\mathfrak{g}}}=$ $2 \pi \eta_{\Sigma}$ and $\eta_{\Sigma}=2|\mathfrak{g}-1|$ for $\mathfrak{g} \neq 1$ and $\eta_{\Sigma}=1$ for $\mathfrak{g}=1$. Such fluxes are subject to opportune Dirac quantization conditions (see $[8,12]$ for details).

So far the discussion has been general, and it can be applied to any 4d SCFT. In [12] an infinite family of $4 \mathrm{~d}$ SCFTs has been considered. This class corresponds to the so called $Y^{p q}$ quiver gauge theories. This notation refers to a family of $\mathrm{SE}_{5}$ manifolds. These manifold have an important application in the AdS/CFT correspondence, because they describe the internal geometry probed by a stack of D3 branes probing the tip of the Calabi-Yau cones over the $Y^{p q}$ manifolds [18].

On the field theory side they correspond to quiver gauge theories, labeled by the positive integer values of $p$ and $q$. For generic values of $p$ and $q$ such theories have four abelian global symmetries. One of such symmetries is the UV R-symmetry $R_{0}$, the others are two mesonic and one baryonic symmetries. In general the mesonic symmetry is $\mathrm{SU}(2) \times$ $\mathrm{U}(1)$ and the baryonic symmetry is $\mathrm{U}(1)$. Further enhancement are possible for some values of $p$ and $q$.

One of the main results of [12] regards the role of the baryonic symmetries in the compactification to two dimensions. In the $4 \mathrm{~d}$ case the exact R-current of the $Y^{p q}$ theories corresponds to a mixing of the abelian mesonic symmetry with $R_{0}$, while the coefficient of the mixing of the baryonic symmetry vanishes. Interestingly, by compactifying to $2 \mathrm{~d}$, the baryonic symmetry can contribute to the exact R-current, i.e. the mixing parameter of such baryonic symmetry is not necessarily vanishing anymore.

One can see this mechanism at work explicitly. Let us turn the flavor symmetries off (i.e. we perform a topological twist just along the R-symmetry and the baryonic symmetry of the $4 \mathrm{~d}$ theory). The field strengths of the $4 \mathrm{~d}$ theory can be written as

$$
F_{R}^{(4 D)} \rightarrow F_{R}^{(2 D)}-\frac{\kappa}{2} t_{\mathfrak{g}}, \quad F_{B}^{(4 D)} \rightarrow F_{B}^{(2 D)}+\epsilon_{B} F_{R}^{(2 D)}-B t_{\mathfrak{g}}
$$

\footnotetext{
${ }^{3}$ One can also twist along non-abelian symmetries along their Cartan subgroup. This procedure breaks such symmetries to $\mathrm{U}(1)$, allowing them to mix with the R-current in two dimensions.
} 


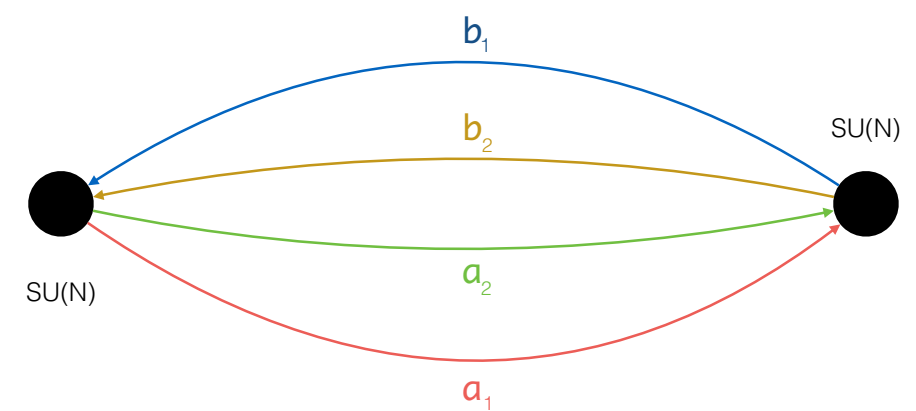

Figure 1. Quiver gauge theory for the Klebanov-Witten theory, describing a stack of D3 branes probing the $Y^{10}=T^{11}$ space.

where $\kappa$ is the normalized curvature of $\Sigma_{\mathfrak{g}}, t_{\mathfrak{g}}$ is the Chern class of the tangent bundle to $\Sigma_{\mathfrak{g}}$ normalized such that $\int_{\Sigma_{\mathfrak{g}}} t_{\mathfrak{g}}=\eta_{\Sigma}[12], B$ is the magnetic flux for the weakly gauged $\mathrm{U}(1)_{B}$ and $\epsilon_{B}$ parameterizes the mixing of $\mathrm{U}(1)_{B}$ with $\mathrm{U}(1)_{R}$.

The $2 \mathrm{~d}$ central charge can be computed in terms of the $4 \mathrm{~d}$ trace anomalies $k_{R R R}$ and $k_{R B B}$ (observe that $k_{B B B}=k_{R R B}=0$ as well as $k_{R}=k_{B}=0$ for $Y^{p q}$ theories). This can be done by starting from the anomalous anomaly polynomial of the $4 \mathrm{~d}$ theory

$$
I_{6}=\frac{k_{R R R}}{6} c_{1}\left(F_{R}\right)^{3}+\frac{k_{R B B}}{6} c_{1}\left(F_{R}\right) c_{1}\left(F_{B}\right)^{2},
$$

where $c_{1}(F)$ is the first Chern class of the bundle with curvature $F$.

Then one has to turn on the background fluxes (2.3) into (2.4) and integrate over $\Sigma_{g}$, obtaining the anomaly polynomial $I_{4}$ of the $2 \mathrm{~d}$ theory. The central charge $c_{2 d}$ corresponds to $k_{R R}$, the coefficient of the term $c_{1}\left(\mathcal{F}_{R}\right)^{2}$ in $I_{4}$. Such central charge will be a function of the mixing coefficient $\epsilon_{B}$. The exact central charge is obtained by extremizing $k_{R R}$ with respect to $\epsilon_{B}$.

Here we consider the case of $p=1$ and $q=0$, corresponding to the Klebanov Witten theory [24]. The associated quiver, represented in figure 1, has two $\mathrm{SU}(N)$ gauge groups connected by two pairs of chiral bifundamentals, $a_{i}$ and two anti-bifundamentals $b_{i}$, with $i=1,2$. This model has a superpotential $W=\epsilon_{i j} \epsilon_{l k} a_{i} b_{l} a_{j} b_{k}$, an R symmetry $\mathrm{U}(1)_{R}$, two $\mathrm{SU}(2)$ flavor symmetries, rotating the matter fields and one baryonic symmetry, assigning opposite charges to the fields $a_{i}$ and $b_{i}$. At the fixed point the exact R-symmetry assigns charge $1 / 2$ to the chiral fields, signaling the absence of mixing between the UV R-current and the other global symmetries.

This theory can be compactified on a Riemann surface along the lines of the discussion above, by turning on a flux $\kappa$ for the R-current and $B$ the baryonic symmetry. The central charge $c_{2 d}$ can be obtained from the knowledge of the anomalies $k_{R R R}$ and $k_{R B B}$. By following the calculation explained above one finds that in this case the mixing parameter is $\epsilon_{B}=2 B / \kappa$ and at large $N$ the central charge is

$$
c_{2 d}=-\eta_{\Sigma} N^{2}\left(\frac{9}{4} \kappa+\frac{12 B^{2}}{\kappa}\right)
$$

and a 2d SCFT can exist only for $\kappa=-1$. 
As a last remark let us consider a twist of a 4d SCFT along the 4d exact R-symmetry only. In this case there is a general relation between $a_{4 d}$ and $c_{2 d}$, first discussed in [5] (see also [12] for further discussions on this "universal twist"). Such relation is

$$
c_{2 d}=-\frac{3 \eta_{\Sigma} \kappa}{2} k_{R R R} N^{2}=-\frac{16 \eta_{\Sigma} \kappa}{3} a_{4 d},
$$

where, again, the last relation shows that $c_{2 d}$ is positive only for $\kappa=-1$.

\section{Flowing from $\mathrm{AdS}_{5}$ to $\mathrm{AdS}_{3}$ in $\mathcal{N}=2$ gauged supergravity}

In this section we discuss some general aspects of the flow across dimensions in gauged supergravity. We start from five dimensional $\mathcal{N}=2 \mathrm{AdS}_{5}$ gauged supergravity with $n_{V}$ vector multiplets (see appendix A for the details on five dimensional supergravity). In this general discussion we do not consider the presence of hypermultiplets, because in some cases they yield to massive vectors, i.e. to broken global symmetries in the SCFT. We return on this problem at the end of the section.

The holographic dictionary identifies the $n_{V}+1$ vectors in the bulk with the R-current (the graviphoton) and $n_{V}$ non-R global currents in the boundary. These vectors can be thought as coming from a consistent truncation of 10D type IIB string theory on $A d S_{5} \times Y_{5}$. In such a case $n_{V}$ represents the sum of some of the internal (mesonic) symmetries of $Y_{5}$ and of some non anomalous baryonic symmetries, corresponding to the Betti vectors in the truncation. The global anomalies on the $\mathrm{AdS}_{5}$ boundary theory are obtained by contracting the sections $X^{I}$ of the Special geometry with the coefficients of the CS terms in the action. The holographic dictionary relates the central charge to the superpotential of the $5 \mathrm{~d}$ theory and $a$-maximization corresponds to the attractor mechanism for the scalars in the Special geometry [30]. The mixing parameters correspond to the coefficients entering in the definition of the graviphoton [30]. The other global anomalies are defined by a similar contraction [31], involving the derivatives ${ }^{4}$ of the sections $X^{I}$.

As mentioned already, the holographic $R G$ flow connects $\mathrm{AdS}_{5}$ vacua with $\mathrm{AdS}_{3} \times \Sigma_{\mathfrak{g}}$ geometries, where $\Sigma_{\mathfrak{g}}$ is a two dimensional Riemann surface with constant curvature and genus $\mathfrak{g}$. This is realized by for solutions of the form

$$
d s_{5}^{2}=e^{2 f(r)}\left(-d t^{2}+d r^{2}+d z^{2}\right)+e^{2 g(r)} d \sigma^{2},
$$

with two radially dependent lapse functions, $f(r)$ and $g(r)$, and area element $\mathrm{d} \sigma^{2}$

$$
d \sigma^{2}=d \mathrm{x}^{2}+F(\mathrm{x})^{2} d \mathrm{y}^{2},
$$

where $F(\mathrm{x})$ is determined by the curvature $\kappa$ (or, alternatively, the genus $\mathfrak{g}$ ) of $\Sigma_{\mathfrak{g}}$ as follows

$$
\left\{\begin{array}{llll}
\mathfrak{g}=0 & \kappa=1 & F(\mathrm{x})=\sin (\mathrm{x}) & x \in[0, \pi], y \in[0,2 \pi[ \\
\mathfrak{g}=1 & \kappa=0 & F(\mathrm{x})=1 & \mathrm{x}, \mathrm{y} \in[0,1[ \\
\mathfrak{g}>1 & \kappa=-1 & F(\mathrm{x})=\sinh (\mathrm{x}) & x \in[0, \infty[
\end{array}\right.
$$

\footnotetext{
${ }^{4}$ This is because the terms $\partial_{i} X^{I}$ appear in $\delta_{\epsilon} \lambda$, and the gauginos correspond to the fermionic components of the vector multiplets dual to the non- $\mathrm{R}$ global currents.
} 
This metric ansatz encompasses both asymptotically locally $\mathrm{AdS}_{5}$ spacetimes and solutions of the form $\mathrm{AdS}_{3} \times \Sigma_{\mathfrak{g}}$, hence the possibility of realizing the flow across dimensions.

The topological twist requires nonvanishing magnetic components of the $n_{V}+1$ field strengths $F^{I}$ of the five dimensional theory. Here we consider a generic ansatz, corresponding to a topological twist along all of the $n_{V}+1$ abelian symmetries of the dual field theory

$$
F_{(5)}^{I}=-a^{I} \operatorname{vol}_{\Sigma_{\mathfrak{g}}}+F_{(3)}^{I}
$$

where the volume form $\operatorname{vol}_{\Sigma_{\mathfrak{g}}}$ is related to $\eta_{\Sigma}$ (introduced above) by $\operatorname{vol}_{\Sigma_{\mathfrak{g}}}=2 \pi \eta_{\Sigma}$ and $a^{I}$ are constant.

By working in units of $R_{A d S_{5}}=1$ the BPS equation relevant for obtaining the $2 \mathrm{~d}$ central charge are $^{5}$

$$
f^{\prime}+e^{f} X^{I} P_{I}+\frac{e^{f-2 g}}{2} a^{I} X_{I}=0, \quad g^{\prime}+e^{f} X^{I} P_{I}-e^{f-2 g} a^{I} X_{I}=0,
$$

together with the constraint on the fluxes $3 P_{I} a^{I}=-\kappa$. The $\mathrm{AdS}_{3}$ fixed point is found by taking $f(r)=f_{0}-\log r$ and constant $g=g^{*}$. In this way the two equations above reduce to a set of algebraic equations. From such algebraic equation we can extract the relations

$$
e^{f_{0}}=\frac{2}{3\left(P^{I} X_{I}\right)}, \quad e^{2 g^{*}}=\frac{a^{I} X_{I}}{P^{I} X_{I}},
$$

and the central charge is obtained from the Brown-Henneaux formula [32]

$$
c_{2 d}=\frac{3 R_{A d S_{3}}}{2 G_{N}^{(3)}} .
$$

The $\mathrm{AdS}_{3}$ radius and the three dimensional Newton constant can be expressed in terms of higher dimensional quantities as

$$
R_{A d S_{3}}=e^{f_{0}}, \quad G_{N}^{(3)}=\frac{G_{N}^{(5)}}{e^{2 g^{*}} \operatorname{vol}_{\Sigma_{g}}}, \quad \text { where } \quad G_{N}^{(5)}=\frac{\operatorname{vol}\left(Y_{5}\right)}{2 \pi^{2} N^{2}}, \quad \operatorname{vol}_{\Sigma_{g}}=2 \pi \eta_{\Sigma} .
$$

By using these relations we arrive at the following expression for the central charge computed from the supergravity data

$$
c_{2 d}=\frac{6 \pi^{3} N^{2} \eta_{\Sigma}}{\operatorname{vol}\left(Y_{5}\right)} e^{f_{0}+2 g^{*}}=\frac{4 \pi^{3} N^{2} \eta_{\Sigma}}{\operatorname{vol}\left(Y_{5}\right)} \frac{a^{I} X_{I}}{\left(P^{I} X_{I}\right)^{2}} .
$$

One can further refine such expression by using the constraint on the Special geometry which relates $X^{I}$ to $X_{I}$. In the normalization that we are considering here the relation is

$$
X_{I}=\frac{1}{6} C_{I J K} X^{J} X^{K}
$$

The R-charges, i.e. the coefficients of the mixing of the global currents in the expression of the R-current, can be read from the graviphoton $A_{\mu}^{I} X_{I}$ [33]: the superconformal Rsymmetry is read off from the anti-commutator of the supercharges acting on the scalar

\footnotetext{
${ }^{5}$ Observe that in this paragraph we use the conventions of [4]. We will modify the conventions in section 5 for making our notation uniform with the literature.
} 
fields, as explained in [27]. ${ }^{6}$ The sections $X_{I}$ are proportional to the mixing coefficients, the R-charges, in the expression of the graviphoton, $r^{I}=q X^{I}$. The correct normalization $q$ on these coefficients can be found by fixing the charge of the gravitino to be one. The gravitino charge is given by the expression $r^{I} P_{I}[30]$, and this fixes the proportionality constant to $t=1 /\left(X^{J} P_{J}\right)$. By considering the relation (3.10) and the definition of the $\mathrm{R}$-charges, the central charge becomes

$$
c_{2 d}=\frac{2 \pi^{3} N^{2} \eta_{\Sigma}}{3 \operatorname{vol}\left(Y_{5}\right)} C_{I J K} a^{I} r^{J} r^{K}
$$

Some comments are in order.

Observe that the structure of the graviphoton exploits the relation between the Rcharges on the field theory side and the R-charges on the gravitational dual description. Consider a SCFT with $n_{V}$ global symmetries in addition to the R-symmetry. In this case we can combine the R-current and the global currents to obtain $n_{V}+1$ trial R-currents, each one charging the superpotential, with non-vanishing R-charge. Let us define these generators as $T_{I}$. The exact $\mathrm{R}$-current in this case is a linear combination of these trial R-currents, $T_{R}=\epsilon_{I} T_{I}$, with the constraint on the mixing parameters $\sum_{I} \epsilon_{I}=2$. The partial topological twist is performed along the generator $T=n_{I} T_{I}$, with $\sum n_{I}=\kappa / 2$. With this choice we can map the generators $T_{I}$ with the vector multiplet gauge fields on the gravity side. The $R$-charges $\epsilon_{I}$ and the fluxes $n_{I}$ are proportional to the charges $r_{I}$ and the fluxes $a_{I}$ discussed on the gravitational side. We will see this mapping explicitly at work in the examples below.

The formulation of $c_{2 d}$ in (3.11) is interesting because it is a quadratic expression in the R-charges, as expected from the field theory side. Moreover the extremization corresponds to an attractor mechanism for the scalars ${ }^{7}$ of the Special geometry, in analogy with the case of $a$-maximization from $\mathrm{AdS}_{5}$ gauged supergravity discussed in [30] and to the case of $3 \mathrm{~d}$ $\tau$-minimization from $\mathrm{AdS}_{4}$ gauged supergravity discussed in [35]. This represents also the $5 \mathrm{~d}$ dimensional version of the result of [36], where the flow from $\mathrm{AdS}_{4}$ to $\mathrm{AdS}_{2}$ in presence of fluxes was studied. In that case the mixing of the graviphoton with the other vector multiplets is related to an attractor mechanism and it is dual to the mixing of the global currents in the exact R-current. This last can be obtained by extremizing the topologically twisted index of [37].

The attractor mechanism can be understood as follows. We have obtained the central charge from gauged supergravity parameterized in terms of a combination of the sections, corresponding to the R-charges. The value of the central charge at the fixed point and the exact mixing are obtained from the analysis of the gaugino variations. Indeed the vector multiplets are associated to the global symmetries mixing with the R-current at the fixed point. At the supersymmetric vacuum the variations lead to the equations (we will provide a detailed derivation in the appendix $\mathrm{B}$ for the case of two vector multiplet, but the result

\footnotetext{
${ }^{6}$ This observation was already made in [30] and allowed the identification of the U(1) R-symmetry in the $5 \mathrm{~d}$ case.

${ }^{7}$ See $[27,34]$ for a complementary discussion.
} 
is more general)

$$
\partial_{x}\left(a^{I} X_{I}\right)-2 e^{2 g} \partial_{x}\left(P_{I} X^{I}\right)=0
$$

where the index $x$ refers to the scalar $\phi^{x}$ in the Special geometry (see appendix A for review). Now we consider the equation (3.9), compute the derivative with respect to the field $\phi^{x}$ : by making use of (3.12) we obtain

$$
\partial_{x} c_{2 d} \propto \frac{1}{\left(X^{I} P_{I}\right)^{2}}\left(\partial_{x}\left(a^{I} X_{I}\right)-2 e^{2 g} \partial_{x}\left(P_{I} X^{I}\right)\right)=0 .
$$

In other words c-extremization corresponds, in gauged supergravity, to the extremization of a superpotential function $W_{3}$ built out of the two quantities $X^{I} P_{I}$, corresponding formally to the superpotential of the $5 \mathrm{~d}$ theory and $a^{I} X_{I}$, corresponding to the central charge of the gauging:

$$
W_{3} \equiv 2 e^{-2 g}\left(e^{-2 g}\left(X_{I} a^{I}\right)-2\left(X^{I} P_{I}\right)\right) .
$$

Lastly, in the case of the "universal twist" there is a relation between $c_{2 d}$ and $a_{4 d}$. In this case one considers a truncation with a graviton (see for example [38] for generic truncations on $Y^{p q}$ manifolds) with $X_{0}=1 /\left(3 X^{0}\right), P_{0}=1 / 3$ and $a^{0}=-\kappa$. By substituting in (3.9) we have

$$
c_{2 d}=\frac{4 \pi^{3} N^{2} \eta_{\Sigma}}{3 \operatorname{vol}\left(Y_{5}\right)}=-\frac{16 \eta_{\Sigma} \kappa}{3} a_{4 d}, \quad \text { with } \quad a_{4 d}=\frac{\pi^{3} N^{2}}{4 \operatorname{vol}\left(Y_{5}\right)}
$$

that requires $\kappa=-1$ and corresponds to the field theory result of formula (2.6).

In the following we apply this formalism to some examples. First we reproduce the results of [8], where $Y_{5}=S^{5}$. Then we discuss the $T^{1,1}$ truncation. In this case we check the result by considering the twist along a combination of the $4 \mathrm{~d}$ exact R-symmetry and the baryonic symmetry. As already mentioned, the $T^{1,1}$ reduction yields a model of (abelian) $\mathcal{N}=25 \mathrm{~d}$ gauged supergravity. This theory is characterized by the presence of a Betti vector multiplet and hypermultiplets as well. The analysis in presence of hypermultiplets is more complicated and in the next paragraph we will discuss its modification.

\subsection{Inclusion of the hypermultiplets}

When considering the hypermultiplets $q^{X}$ there can be massive vector multiplets in the spectrum. ${ }^{8}$ On the supergravity side the condition for massive vectors is $K_{I}^{X} X^{I}=0$ (see for example [39]). As reviewed in [30], by expanding the prepotentials around the origin one has, at linearized level, $P_{I}=P_{I(0)}+m_{I}^{a} P_{a}$, where $P_{a}$ plays the role of a Lagrange multiplier. The holographic dictionary associates these massive vector bosons to anomalous global currents. Their contribution can be added to $a_{4 d}$ by introducing the multiplier: this sets a constraint preventing the massive vectors to mix with the graviphoton.

Turning on the fluxes and flowing to the $\mathrm{AdS}_{3}$ vacuum in general triggers the flow of the hyperscalars as well. Nevertheless at the $\mathrm{AdS}_{3}$ fixed point the constraint on the massive vector needs to be satisfied as well. By enforcing the constraint in the relation

\footnotetext{
${ }^{8}$ Observe that it is not always the case, for example consistent truncations with Betti hypermultiplets turned do not higgs any further symmetries and do not lead to massive vectors.
} 
between the sections in the $\mathrm{AdS}_{3}$ vacuum we forbid the mixing of the massive vector with the graviphoton. As we will see in detail later, at the $\mathrm{AdS}_{3}$ point a further constraint on the fluxes $a^{I}$ needs to hold. Such constraint, coming from the BPS equations, has to be imposed to recover the structure of the topological twist. Physically speaking this corresponds to the gravity dual mechanism of a consistency requirement for the topological twist, i.e. on the field theory side one can perform the topological twist only along conserved non anomalous global abelian currents.

\section{$4 \mathcal{N}=4 \mathrm{SYM}$ and $\mathrm{U}(1)^{3}$ gauged supergravity}

As a first example we reproduce the results of [8], namely we compute the two dimensional central charge obtained by the R-symmetry twist of $4 \mathrm{~d} \mathcal{N}=4 \mathrm{SYM}$ to $2 \mathrm{~d} \mathcal{N}=(0,2)$. In this case there is an $\mathrm{SO}(6)_{R}$ symmetry and the twist is done along the $\mathrm{SO}(2)_{R}^{3}$ abelian subgroup, i.e.

$$
T=a^{I} T_{I},
$$

where $T_{I}$ are the generators of the abelian symmetries and $a^{I}$ correspond to the magnetic fluxes of the weakly gauged abelian factors. ${ }^{9}$ If $\sum a^{I}=-\kappa$ and for generic $a^{I}$, supersymmetry is broken to $\mathcal{N}=(0,2)$ [8]. The $2 \mathrm{~d}$ R-current $T_{R}$ is a linear combination of the three abelian symmetries $T_{R}^{(2 d)}=\epsilon^{I} T_{I}$, where the constraint $\sum \epsilon^{I}=2$ has to be enforced. The central charge $c_{2 d}$ has been computed in [12] as a function of such mixing. This has been extremized in terms of $\epsilon^{I}$. At the fixed point such mixing parameters are

$$
\epsilon^{I}=\frac{2 a^{I}\left(2 a^{I}+\kappa\right)}{\theta} \quad \text { with } \quad \theta \equiv 2 \sum_{I=1}^{3}\left(a^{I}\right)^{2}-\left(\sum_{I=1}^{3} a^{I}\right)^{2}
$$

and the central charge becomes

$$
c_{2 d}=-12 \eta_{\Sigma} N^{2} \frac{a^{0} a^{1} a^{2}}{\theta} .
$$

In what follows we want to reproduce this result in the gauged supergravity setup. In this case $Y_{5}=S^{5}$ and we use the conventions of [8], i.e.

$$
R_{A d S_{5}}=1, \quad P_{I}=\frac{1}{3}, \quad 3 X_{I}=\left(X^{I}\right)^{-1} .
$$

The constraint on the fluxes is $3 a^{I} P_{I}=\sum a^{I}=-\kappa$ and we define the R-charges as $r^{I}=X^{I} /\left(X^{J} P_{J}\right)$. By plugging in these values in (3.11) the central charge is

$$
c_{2 d}=\frac{4}{3} N^{2} \eta_{\Sigma}\left(a^{0} r^{1} r^{2}+a^{1} r^{2} r^{0}+a^{2} r^{0} r^{1}\right)
$$

We extremize this function with the constraint on the R-charges $\sum r^{I}=3$. At the fixed point the charges are

$$
r^{0}=\frac{3 a^{0}}{\theta}\left(a^{0}-a^{1}-a^{2}\right), \quad r^{1}=\frac{3 a^{1}}{\theta}\left(a^{1}-a^{0}-a^{2}\right)
$$

and the central charge matches the field theory result (4.2) for $\kappa=-1$.

\footnotetext{
${ }^{9}$ Observe that here we are keeping the same notations for the indices that we adopted on the supergravity side, even if there is no meaning in rising and lowering the index $I$ in field theory. Here the index $I$ runs over the Cartan subgroup of the global symmetry group.
} 


\section{$5 \quad T^{1,1}$ with a Betti multiplet}

In this section we discuss the main result of our paper. We study a consistent $\mathcal{N}=2$ truncation of $T^{1,1}$ with a Betti multiplet [25] and we reproduce the mixing of this vector with the graviphoton of the $5 \mathrm{~d}$ theory when flowing to $\mathrm{AdS}_{3}$. Let us first recall some general aspects of the baryonic symmetries and their relation with the Betti vector multiplets. We keep the discussion general so far. When considering the KK spectrum of IIB supergravity on $\mathrm{AdS}_{5} \times \mathrm{SE}_{5}$ one can see the emergence of new massless matter arising from the non trivial second cohomology group of the $\mathrm{SE}_{5}$ manifold. In general a truncation to $5 \mathrm{~d} \mathcal{N}=4$ SUGRA yields $b_{3}\left(\mathrm{SE}_{5}\right)$ of such vector multiplets, where $b_{3}$ is the third Betti number of $\mathrm{SE}_{5}$. Such $\mathcal{N}=4$ vector multiplets, called Betti vectors, can be further truncated to $\mathcal{N}=2$ vector multiplets or $\mathcal{N}=2$ Betti hypermultiplets. The former are dual to non anomalous conserved baryonic currents [40] (which are those we consider) while the latter are associated to exactly marginal operators.

We will be interested to truncation leaving the Betti vector multiplets in the spectrum. ${ }^{10}$ Such a truncation has been explicitly obtained for the case of $T^{1,1}$ in [25] (see also [41]). In this case $\mathrm{b}_{3}\left(T^{1,1}\right)=1$, i.e. there is a single Betti vector and it is dual to the baryonic symmetry of the Klebanov Witten theory. On the field theory side this baryonic symmetry can be identified as follows. The model has two $\mathrm{U}(N)$ gauge groups. In the IR the dynamical gauge groups is $\mathrm{SU}(N)^{2}$, i.e. the two $\mathrm{U}(1)$ symmetry become flavor symmetries, one of which is redundant. The leftover $\mathrm{U}(1)$ corresponds to the baryonic symmetry of interest. Such symmetry does not mix with the R-charge in $4 \mathrm{~d}$. This is a general statement, see for example [22] for a general discussion.

The $\mathcal{N}=2$ truncation of [25] has a third vector, which is Higgsed due to the presence of extra hypermultiplets, containing a Stuckelberg field. ${ }^{11}$ This requires some modification to our general discussion, along the lines of section (3.1).

The section is organized as follows. First we introduce the details of the truncation in $5 \mathrm{~d}$, then we study the $\mathrm{AdS}_{3}$ vacuum. We perform the numeric analysis to study the holographic RG flow from the $\mathrm{AdS}_{5} \mathrm{UV}$ fixed point to the $\mathrm{AdS}_{3} \mathrm{IR}$ one. We conclude by computing $c_{2 d}$ and the structure of the mixing of the global currents of the $2 \mathrm{~d}$ theory with the formalism developed above, matching the result with the one obtained in field theory. Let us finally mention that BPS solutions interpolating between an $\mathrm{AdS}_{4}$ UV fixed point and $\mathrm{AdS}_{2}$, in theories with hypermultiplets and Betti multiplets have recently been found in [42].

\subsection{The 5d truncation}

Here we review the truncation of $10 \mathrm{~d}$ type IIB on $T^{1,1}$ leading to $5 \mathrm{~d}$ gauged supergravity with a Betti vector, the so-called "Betti-vector" truncation of [25], subsequently studied in [41]. We refer the reader to these articles for further details. This model consists of the $\mathcal{N}=2$ graviton multiplet, two $\mathcal{N}=2$ vector multiplets and two hypermultiplets (one of which is the universal hypermultiplet), and it admits a $\mathcal{N}=2$ supersymmetric $\mathrm{AdS}_{5}$ vacuum.

\footnotetext{
${ }^{10}$ See [9] for a reduction to $\mathrm{AdS}_{3}$ with the Betti hypermultiplet turned on.

${ }^{11}$ See for example [39] for a general discussion on such higgsing in gauged supergravity.
} 
Schematically, the bosonic matter content is [41]

- gravity +2 vectors: $\left(g_{\mu \nu}, A_{0}, A_{1}, A_{2}, u_{2}, u_{3}\right)$;

- 2 hypers: $\left(u_{1}, k, \tau, \bar{\tau}, b_{0}^{i}, \bar{b}_{0}^{i}\right), i=1,2$.

In this truncation one of the vectors becomes massive via Higgs mechanism, and this vector multiplet plus one hypermultiplet become a massive vector multiplet. The matter content can also be rewritten in the following way (table 2 of [25]):

- the gravity multiplet, containing a graviton $g_{\mu \nu}$ and a massless vector $A_{g}$;

- A massless Betti vector multiplet, containing a massless vector $A_{\mathcal{B}}$ and one real scalar $u_{2}$ with $m^{2}=-4(\Delta=2)$;

- A universal hypermultiplet, with 4 real fields $\tau, \bar{\tau}, b_{0}^{1}, \bar{b}_{0}^{1}$, with $m_{\tau}^{2}=0(\Delta=4)$ and $m_{b_{0}}^{2}=-3(\Delta=3)$

- A massive vector multiplet, containing a massive vector $A_{m}$ (who has eaten its axion $k$ ) with $m^{2}=24, \Delta=7$, and 4 real scalars $u_{3}, u_{1}, b_{0}^{2}, \bar{b}_{0}^{2}$ with $m^{2}=12,21,21,32$ $(\Delta=6,7,7,8)$.

The quaternionic Killing vectors, specifying the gauging, are:

$$
K_{0}=\left(3 i b_{0}^{i} \partial_{b_{0}^{i}}+\text { c.c. }\right)-2 \partial_{k}, \quad K_{1}=2 \partial_{k}, \quad K_{2}=2 \partial_{k}
$$

and the Killing prepotentials are

$$
\begin{aligned}
& P_{0}=-i\left[\left(3-\frac{1}{2} e^{-4 u_{1}} e^{Z}\right) \sigma_{3}-2 i e^{-2 u_{1}} v_{i} f_{0}^{i} \sigma_{+}+2 i e^{-2 u_{1}} \bar{v}_{i} \bar{f}_{0}^{i} \sigma_{-}\right] \\
& P_{1}=-i e^{-4 u_{1}} \sigma_{3} \\
& P_{2}=-i e^{-4 u_{1}} \sigma_{3}
\end{aligned}
$$

where $v_{i}$ is the $\mathrm{SL}(2, R)$ vielbein and

$$
f_{0}^{i}=3 i b_{0}^{i}, \quad e^{Z}=4-\frac{2 i}{3} \epsilon_{i j}\left(f_{0}^{i} \bar{f}_{0}^{j}-\bar{f}_{0}^{i} \cdot f_{0}^{j}\right) .
$$

We can moreover express the vectors $A^{0}, A^{1}, A^{2}$ appearing in the model in terms of the Betti vector $A_{\mathcal{B}}$, the massless vector appearing in $\mathrm{SE}_{5}$ truncation $A_{g}$ and the massive vector $A_{m}$, in this way

$$
\begin{aligned}
& A_{0}=\frac{1}{3}\left(-\sqrt{6} A_{m}+\sqrt{3} A_{g}\right), \\
& A_{1}=\frac{1}{6}\left(\sqrt{6} A_{m}-3 \sqrt{2} A_{\mathcal{B}}+2 \sqrt{3} A_{g}\right), \\
& A_{2}=\frac{1}{6}\left(\sqrt{6} A_{m}+3 \sqrt{2} A_{\mathcal{B}}+2 \sqrt{3} A_{g}\right),
\end{aligned}
$$


the inverse relations being

$$
A_{\mathcal{B}}=\frac{A_{2}-A_{1}}{\sqrt{2}}, \quad A_{m}=\frac{A_{1}+A_{2}-2 A_{0}}{\sqrt{6}}, \quad A_{g}=\frac{A_{0}+A_{1}+A_{2}}{\sqrt{3}} .
$$

One can see that when $A^{2}=A^{1}$ the Betti vector vanishes, and, upon a suitable truncation of the scalars, we are back to the $\mathrm{SE}_{5}$ truncation. The expressions we just wrote will be useful when dealing with the explicit form of the mixing for the baryonic symmetries in the R-symmetry.

Let us finally mention that the $\mathrm{AdS}_{5}$ vacuum is obtained for the following values of the scalar fields:

$$
u_{1, A d S_{5}}=u_{2, A d S_{5}}=u_{3, A d S_{5}}=b_{0, A d S_{5}}^{1}=b_{0, A d S_{5}}^{2}=\tau=0,
$$

\subsection{The ansatz and the BPS equations}

We study now the flow from $\mathrm{AdS}_{5}$ to $\mathrm{AdS}_{3} \times \Sigma_{g}$ by solving the BPS equations. We consider the metric ansatz discussed in section 3 , which we rewrite here for convenience:

$$
d s_{5}^{2}=e^{2 f(r)}\left(-d t^{2}+d r^{2}+d z^{2}\right)+e^{2 g(r)} d \sigma^{2} .
$$

The gauge fields read

$$
\left\{\begin{array}{lll}
\mathfrak{g}=0 & \kappa=1 & A_{\mathrm{x}}^{I}=0, A_{\mathrm{y}}^{I}=a^{I} \cos (\mathrm{x}) \\
\mathfrak{g}=1 & \kappa=0 & A_{\mathrm{x}}^{I}=-\frac{1}{2} a^{I} \mathrm{y}, A_{\mathrm{y}}^{I}=\frac{1}{2} a^{I} \mathrm{x} \\
\mathfrak{g}>1 & \kappa=-1 & A_{\mathrm{x}}^{I}=0, A_{\mathrm{y}}^{I}=-a^{I} \cosh (\mathrm{x})
\end{array}\right.
$$

hence the field strengths, defined as $F_{\mu \nu}^{I}=\partial_{\mu} A_{\nu}^{I}-\partial_{\nu} A_{\mu}^{I}$ read

$$
F^{I}=-a^{I} F(\mathrm{x}) d \mathrm{x} \wedge d \mathrm{y} \quad I=0,1,2 .
$$

We will be interested here in the purely magnetic case. ${ }^{12}$ The scalar fields of the Special geometry are positive, satisfy $X^{0} X^{1} X^{2}=1$ and they are parameterized as

$$
X^{0}=e^{4 u_{3}}, \quad X^{1}=e^{2 u_{2}-2 u_{3}}, \quad X^{2}=e^{-2 u_{2}-2 u_{3}}, \quad X_{I}=\left(X^{I}\right)^{-1} .
$$

The two real scalars $u_{2}$ and $u_{3}$ are independent and belong to the vector multiplets.

We will make one simplifying assumption while looking for solutions of the BPS equations. First of all, as shown in [25], one can consistently truncate away the universal hypermultiplet fields. Hence $\tau, b_{0}^{1}$ will be set to constant values. Moreover, the remaining fields $b_{0}^{2}$ appears quadratically in the action, hence can be consistently se to zero. This setup was shown to be a consistent truncation in [43] as well, the field $u_{1}$ being called the resolution mode of $T^{1,1} \cdot{ }^{13}$

\footnotetext{
${ }^{12} t$ and $z$ components of the gauge fields of the form $A_{t}^{I}=q(r)$ and $A_{z}^{I}=w^{I}=$ const are compatible with the Maxwell and Bianchi equations as well, and contribute to the black string electric charge $\int_{\Sigma_{g}} G_{I}$, where $G_{I, \mu \nu}=\epsilon_{\mu \nu}{ }^{\sigma \rho} \frac{\partial \mathcal{L}}{\partial F_{I}^{\sigma \rho}}$. We will not consider them here.

${ }^{13}$ Our Lagrangian is the same as (7.7) of [43] upon the following field identification

$$
\eta=\frac{2\left(2 u_{1}+3 u_{3}\right)}{5}, \quad \chi=\frac{2}{5}\left(3 u_{1}-3 u_{3}\right), \quad \lambda=2 u_{2}
$$


We will then look for explicit solutions where all the scalar fields except $u_{1}, u_{2}, u_{3}$ are consistently set to zero. One can see from eq. (5.2) that this has the net effect of setting the components $r=1,2$ of the Quaternionic Killing prepotentials $P_{\Lambda}^{r}$ to zero:

$$
P_{\Lambda}^{1}=P_{\Lambda}^{2}=0,
$$

namely in our solutions, similarly to [42], the prepotentials are aligned in the $\sigma^{3}$ direction, namely $P_{I}^{3} \equiv-i P_{I} \neq 0$. Moreover, this has the net effect of setting $\omega_{\mu i}^{j}=0$.

The full analysis of the supersymmetry variations is in appendix B, where the BPS equations are obtained. In the case under consideration $Q^{r}=(0,0-1)$, hence the projection on the Killing spinor simplifies to

$$
\gamma_{2} \epsilon_{i}=\sigma^{3}{ }_{i j} \epsilon^{j}, \quad \gamma_{34} \epsilon_{i}=i \sigma^{3}{ }_{i j} \epsilon^{j},
$$

and the BPS equations then reduce to:

$$
\begin{aligned}
f^{\prime}+\frac{1}{3} e^{f} X^{I} P_{I}+\frac{1}{6} a^{I} X_{I} e^{-2 g+f} & =0 \\
g^{\prime}+\frac{1}{3} e^{f} X^{I} P_{I}-\frac{1}{3} a^{I} X_{I} e^{-2 g+f} & =0 \\
8 u^{2^{\prime}}+\frac{e^{-2 g+f}}{2} a^{I} \partial_{2} X_{I}-e^{f} \partial_{2} X^{I} P_{I} & =0 \\
24 u^{3^{\prime}}+\frac{e^{-2 g+f}}{2} a^{I} \partial_{3} X_{I}-e^{f} \partial_{3} X^{I} P_{I} & =0 \\
16 u_{1}^{\prime}-2 \partial_{X}\left(X^{I} P_{I}\right) & =0 \\
a^{I} K_{I}^{X} & =0 \\
a^{I} P_{I}+\kappa & =0
\end{aligned}
$$

where we used the fact that $g_{22}=8, g_{33}=24, g_{23}=0$ and the metric of the hypermultiplet $u_{1}$ is $g_{11}=16$. Lastly, one can see that the BPS equations (5.14)-(5.14) select a particular value for the Dirac quantization conditions of the theory, correspondingly, on the field theory side, to the quantization of the fluxes through the Riemann surface.

\subsection{The $\mathrm{AdS}_{3}$ vacuum}

In order for the metric to approach the $\mathrm{AdS}_{3} \times \Sigma_{\mathfrak{g}}$ fixed point, the metric functions should attain the constant value $f(r)=f_{0}-\log r$ and $g=g *$. The scalars as well assume constant values, which we denote by $\phi_{X}^{*}$ and $q_{i}^{*}$. At the $\mathrm{AdS}_{3}$ vacuum the equations (5.14) reduce to a set of algebraic equations for $g^{*}, f_{0}$ and the scalars

$$
\begin{aligned}
2 e^{2 g^{*}}\left(X^{I} P_{I}\right) & =C_{I J K} a^{I} X^{J} X^{K} \\
e^{f_{0}}\left(X^{I} P_{I}\right) & =2 \\
2 e^{2 g^{*}}\left(X^{1} P_{1}-X^{2} P_{2}\right) & =X^{0}\left(a^{2} X^{1}-a^{1} X^{2}\right) \\
2 e^{2 g^{*}}\left(2 X^{0} P_{0}-X^{1} P_{1}-X^{2} P_{2}\right) & =a^{1} X^{0} X^{2}+a^{2} X^{0} X^{1}-2 a^{0} X^{1} X^{2}
\end{aligned}
$$


with the constraints

$$
a^{0}=-\kappa / 3, \quad a^{1}+a^{2}=2 a^{0}, \quad X^{1}+X^{2}=2 X^{0} .
$$

Notice that in order to obtain (5.15) and (5.16) the first one of the projections (5.13) becomes unnecessary. In other words, while the entire flow preserves one quarter of supersymmetries, the supersymmetry is actually enhanced to $1 / 2$ BPS at the near-horizon $\mathrm{AdS}_{3} \times \Sigma_{\mathfrak{g}}$ geometry.

Let's now analyze the equations (5.15) and (5.16) obtained. The first two relations reproduce, in gauged supergravity, the structure of fluxes turned on when performing the topological twist on the field theory side. Here we parameterize the fluxes $a^{1}$ and $a^{2}$ as

$$
a^{1}=-\frac{1}{3}(\kappa-4 b), \quad a^{2}=-\frac{1}{3}(\kappa+4 b) .
$$

The last constraint in (5.16), corresponding to $K_{X}^{I} X_{I}=0$, signals the presence of a massive vector multiplet in the spectrum, i.e. a non conserved abelian current on the field theory side. This is consistent with the fact that there are only two independent conserved charges $a^{I}$. In the case of $\kappa=-1$ the $\operatorname{AdS}_{3}$ vacuum is found at

$$
\begin{aligned}
& e^{4 u_{1}}=\frac{3 \kappa^{2}+16 b^{2}}{3 \kappa^{2}}, \quad \quad e^{4 u_{2}}=\frac{\kappa+4 b}{\kappa-4 b}, \quad e^{12 u_{3}}=\frac{\kappa^{2}}{\kappa^{2}-16 b^{2}}, \\
& e^{6 g^{*}}=\frac{\left(16 b^{2}+3 \kappa^{2}\right)^{3}}{729 \kappa\left(16 b^{2}-\kappa^{2}\right)}, \quad e^{3 f_{0}}=\frac{8}{27} \frac{\kappa^{2}-16 b^{2}}{\kappa^{2}} .
\end{aligned}
$$

At this point one is ready to compute the central charge, by plugging in the values of $g^{*}$ and $f_{0}$ in (5.18) in the Brown-Henneaux formula (3.7):

$$
c_{2 d}=-\eta_{\Sigma} N^{2}\left(\frac{9 \kappa}{4}+\frac{12 b^{2}}{\kappa}\right) .
$$

This shows that only solutions with $\kappa=-1$ are admitted, as expected from the field theory side. Indeed the case $\kappa=0$ is singular, while the case $\kappa=1$ gives a negative central charge. This match with the central charge obtained on the field theory side (2.5).

\subsection{Holographic RG flow}

Here we numerically solve the BPS flow equations, studying the holographic RG flow connecting the $\mathrm{AdS}_{5} \mathrm{UV}$ vacuum and the $\mathrm{AdS}_{3}$ IR one. In order to simplify the numerical problem we rewrite the BPS equations in an easier and more compact form by a change of variables.

This change of variables is analogous to the one discussed in $[8,44]$. First one start by redefining the radial coordinate as

$$
\rho \equiv f+2 g
$$

The second step consists in considering a superpotential function ${ }^{14}$ for the theory on $\mathrm{AdS}_{3}$. This corresponds to a combination of the $\mathrm{AdS}_{5}$ superpotential $X^{I} P_{I}$ discussed in [41] and of

\footnotetext{
${ }^{14}$ This is the same superpotential that we already encountered in section 3 when we observed that cextremization corresponds to an attractor mechanism in gauged supergravity. This is possible because, even if we are in presence of hypermultiplets, we can still rotate the killing potentials $P_{I}$ along the same direction.
} 

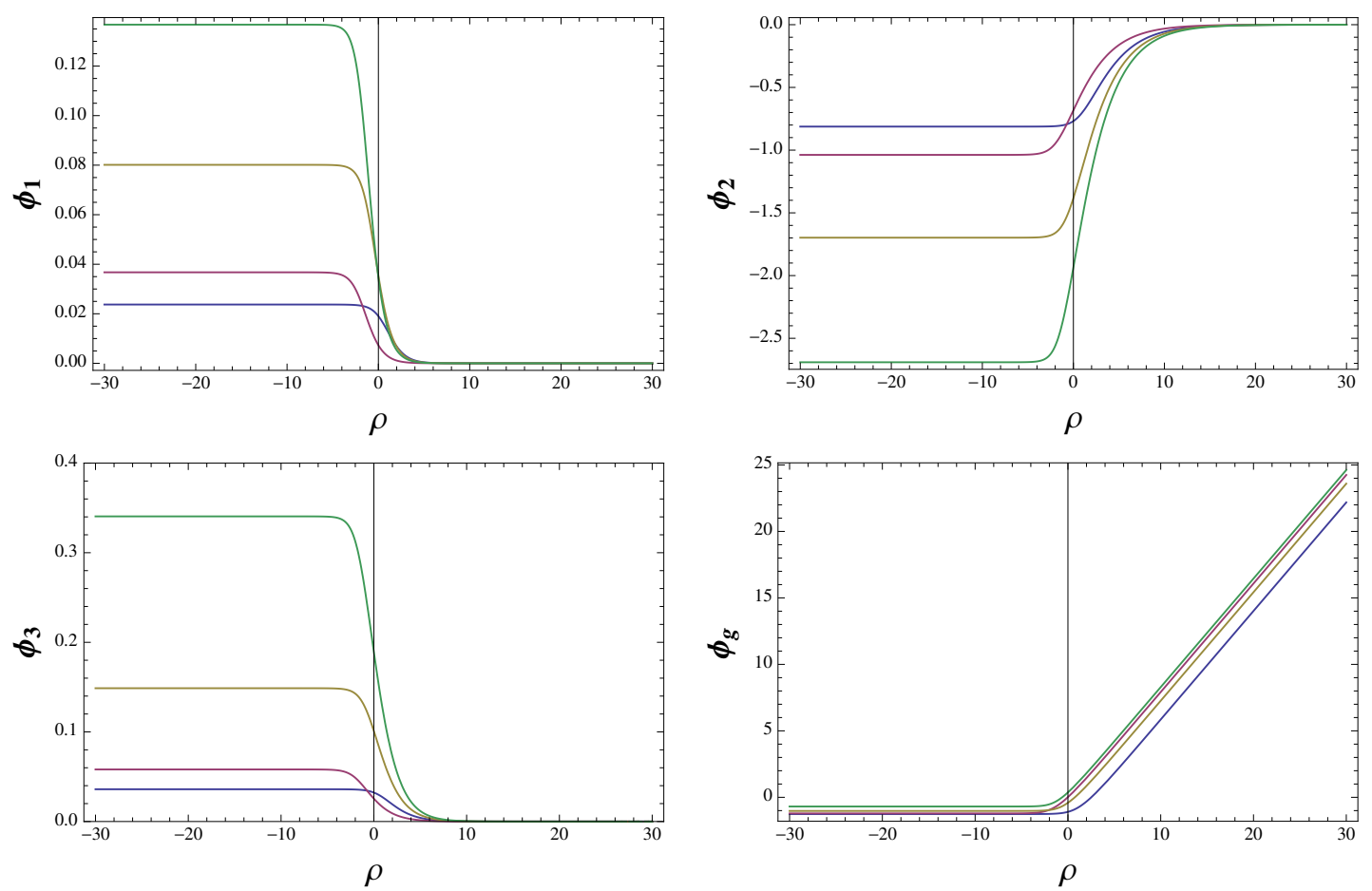

Figure 2. Holographic RG flow for the of the scalar fields $\phi_{i}$ interpolating between the $\mathrm{AdS}_{5}$ vacuum in the UV, $\rho \rightarrow \infty$ and the $\mathrm{AdS}_{3}$ vacuum in the IR, $\rho \rightarrow-\infty$. The colors refers to the values $b=0.08$ (blue), $b=0.1$ (red), $b=0.15$ (yellow) and $b=0.2$ (green).

the central charge of the gauging $X_{I} a^{I}$. Their combination give rise to the $3 \mathrm{~d}$ superpotential function (3.14), that can be used to reformulate the BPS equation in a compact form. The BPS equations for $f^{\prime}$ and $g^{\prime}$ can be recombined in

$$
\frac{\partial \rho(r)}{\partial r}=\frac{e^{\rho}}{2} W_{3}
$$

The other BPS equations can be written as

$$
\frac{\partial u_{i}}{\partial r}=-\frac{1}{2} e^{\rho} g^{i i} \partial_{i} W_{3}, \quad \frac{\partial g(r)}{\partial r}=-\frac{e^{\rho}}{12} \frac{\partial W_{3}}{\partial g} .
$$

These equations are more suitable for the numerical analysis, and they are independent of $f$. Once we have the solution for these equations the function $f$ is determined automatically from (5.21). Moreover, by using (5.21), the equations (5.22) become

$$
\frac{\partial u_{i}}{\partial \rho}=-g^{i i} \frac{1}{W_{3}} \frac{\partial W_{3}}{\partial u_{i}}, \quad \frac{\partial g}{\partial \rho}=-\frac{1}{6 W_{3}} \frac{\partial W_{3}}{\partial g} .
$$

It follows that if we canonically normalize the fields $u_{i}$ by $\sqrt{g}$ ii and $g$ by $\sqrt{6}$, and define a new set of variables $\phi_{1}, \phi_{2}, \phi_{3}$ and $\phi_{g}$ the BPS equations can be written together as

$$
\frac{\partial \phi_{i}}{\partial \rho}=-\frac{1}{W_{3}} \frac{\partial W_{3}}{\partial \phi_{i}} .
$$


This formulation shows that a slight simplification appears when working in $3 \mathrm{~d}$ versus $5 \mathrm{~d}$, because the $3 \mathrm{~d}$ theory appears to be agnostic of the distinction between scalars from hypermultiplets and vector multiplets. This fact was noticed in [45] for the model treated in section 6 (see related discussion in that section). Indeed, one sees that the BPS flow equations are generated by varying a single superpotential function, which in the present case is denoted by $W_{3}$.

Coming back to our analysis, in this basis the $\mathrm{AdS}_{3}$ vacuum corresponds to

$$
\begin{aligned}
\phi_{1} & =\sqrt{\frac{1}{2}} \log \left(\frac{16 b^{2}+3 \kappa^{2}}{3 \kappa^{2}}\right), & \phi_{2} & =\sqrt{\frac{3}{2}} \log \left(\frac{\kappa+4 b}{\kappa-4 b}\right), \\
\phi_{3} & =\frac{1}{3} \log \left(\frac{\kappa^{2}}{\kappa^{2}-16 b^{2}}\right), & \phi_{g} & =\frac{1}{\sqrt{6}} \log \left(\frac{\left(16 b^{2}+3 \kappa^{2}\right)^{3}}{729 \kappa\left(16 b^{2}-\kappa^{2}\right)}\right) .
\end{aligned}
$$

We can now numerical solve these equations by fixing $\kappa=-1$ and by imposing the boundary conditions in the IR, i.e. the fact the solution flows to the $\mathrm{AdS}_{3}$ vacuum in formula (5.25). From the figure 2 we can observe that at large $\rho$ this theory flows to a different fixed point, in which the fields $\phi_{i}$ sit at the $\mathrm{AdS}_{5}$ vacuum (5.6).

\section{5 c-extremization from gauged supergravity}

In this section we are interested in the structure of the mixing of the abelian currents leading to $c_{2 d}$ as a result of the extremization procedure. We study the $c$-extremization in gauged supergravity along the lines of section 3. Interestingly we see that the effect of the hypermultiplets can be easily included here by enforcing the constraint on the massive vector. This can be done by parametrizing the mixing in terms of the R-charges $r^{I}$ and imposing the constraint $2 X_{0}=X_{1}+X_{2}$. In this way the R-charges become

$$
r^{0}=\frac{1}{3}, \quad r^{1}=\frac{X^{1}}{3 X^{0}}=\frac{1}{3}(1-\epsilon), \quad r^{2}=\frac{X^{2}}{3 X^{0}}=\frac{2 X^{0}-X^{1}}{3 X^{0}}=\frac{1}{3}(1+\epsilon) .
$$

where we defined $\epsilon=-\left(X^{1}-X^{0}\right) / X^{0}$. We reproduce the topological twist by identifying the R-symmetry and the baryonic symmetry with the graviphoton and with the Betti vector respectively. Explicitly

$$
a^{I} T_{I}=\frac{k}{3}\left(T_{0}+T_{1}+T_{2}\right)+\frac{4}{3} b\left(T_{2}-T_{1}\right)
$$

hence the identifications between the field theory generators $T_{R}^{f . t .}$ and $T_{B}^{f . t .}$ and the supergravity ones

$$
T_{R}^{f . t .}=\frac{3}{2}\left(T_{0}+T_{1}+T_{2}\right), \quad T_{B}^{f . t .}=\frac{3}{4}\left(T_{2}-T_{1}\right) .
$$

This decomposition reflects eq. (5.5). By using the relation $\operatorname{vol}\left(T^{1,1}\right)=\frac{16 \pi^{3}}{27}$ the central charge can be written as ${ }^{15}$

$$
c_{2 d}=\frac{6 \pi^{3} N^{2} \eta_{\Sigma}}{\operatorname{vol}\left(T^{1,1}\right)} C_{I J K} a^{I} r^{J} r^{K}=\frac{3 N^{2} \eta_{\Sigma}}{4}\left(8 b \epsilon-\kappa\left(3-\epsilon^{2}\right)\right) .
$$

\footnotetext{
${ }^{15}$ Observe that in our conventions $2 X_{I}=C_{I J K} X^{J} X^{K}$ and $e^{f}=2 /\left(X^{I} P_{I}\right)$.
} 
At the extremum, corresponding to $\epsilon^{*}=-4 b / \kappa$, the central charge becomes

$$
c_{2 d}=-\eta_{\Sigma} N^{2}\left(\frac{9 \kappa}{4}+\frac{12 b^{2}}{\kappa}\right)
$$

matching the field theory result (2.5) for $\kappa=-1$. Observe that as a consistency check we can plug in $c_{2 d}$ the values of the scalars at the vacuum (5.18) and obtain the value of $\epsilon^{*}$.

\section{$6 \quad$ M5 branes}

In this section we discuss the flow to $\mathrm{AdS}_{3}$ for a $5 \mathrm{~d}$ theory constructed in [46] from a consistent truncation of $7 \mathrm{~d} \mathcal{N}=4 \mathrm{SO}(5)$ gauged supergravity reduced on a Riemann surface. The holographic $4 \mathrm{~d}$ theories associated to such truncation were constructed in [28]. They are quiver gauge theories, obtained from the class $\mathrm{S}$ theories of [47] by gluing the pants such that only $\mathcal{N}=1$ supersymmetry is preserved. We will not review the construction here, and refer the reader to the original paper for details. ${ }^{16}$ We do not discuss the calculation of the central charge from the field theory data (see [10]) and the analysis of the flow in gauged supergravity (see $[8,10])$. Here we are just interested in constructing $c_{2 d}$ by applying our formalism. We can also check our result against the one obtained in [8], where the reduction of the $7 \mathrm{~d}$ theory on the product of two Riemann surfaces $\Sigma_{1} \times \Sigma_{2}$ was discussed.

By using our formalism, $c_{2 d}$ can be obtained from $5 \mathrm{~d} \mathcal{N}=2$ gauged supergravity in terms of the prepotentials $P_{I}$. The supergravity analysis leads to the following Killing prepotentials $^{17}$

$$
P_{0}=e^{u_{3}}, \quad P_{1}=2+e^{u_{3}} \frac{z_{1}+\kappa_{1}}{8}, \quad P_{2}=2-e^{u_{3}} \frac{z_{1}-\kappa_{1}}{8} .
$$

The sections are parameterized as

$$
X^{0}=e^{u_{1}+u_{2}}, \quad X^{1}=e^{-u_{1}}, \quad X^{2}=e^{-u_{2}} .
$$

By following the procedure discussed above we can reduce this theory on $\Sigma_{2}$, with curvature $\kappa_{2}$. The constraints on the fluxes are

$$
2 a^{1}+2 a^{2}=-\kappa_{2} \quad 8 a^{0}+a^{1}\left(\kappa_{1}-z_{1}\right)+a^{2}\left(\kappa_{1}+z_{1}\right)=0 .
$$

We can parameterize $a^{1}$ and $a^{2}$ as

$$
a^{1}=-\frac{\kappa_{2}-z_{2}}{4}, \quad a^{2}=-\frac{\kappa_{2}+z_{2}}{4},
$$

where $z_{2}$ plays the role of the baryonic symmetry discussed in the case of $T^{1,1}$, i.e. it is associated to a massless vector multiplet that does not mix with the $5 \mathrm{~d}$ graviphoton, but

\footnotetext{
${ }^{16}$ This model was discussed in [45]. In the same paper the uplift in $10 \mathrm{~d}$ is constructed and the authors showed that the R-symmetry agrees with the canonical R-symmetry in 11D based on the existing classification, [17].

${ }^{17}$ We are modifying the notations of $[46]$, with the change of variables $\lambda_{1}=\frac{-4 u_{1}+u_{2}-u_{3}}{10}, \lambda_{2}=$ $\frac{u_{1}-4 u_{2}+u_{3}}{10}, B=\frac{3\left(u_{1}+u_{2}-u_{3}\right)}{10}$.
} 
that can mix in 3d. One can observe that $a^{1}+a^{2}=-\kappa_{2} / 2$ and $a^{1}-a^{2}=z_{2} / 2$ are the fluxes for the $5 \mathrm{~d}$ graviphoton, that can be obtained from $a$-maximization, and for the second vector multiplet, that couples in the two dimensional CFT as expected. In this case there is a massive vector multiplet as well. Its effect can be understood from the hyperino variation, constraining the scalars as

$$
8 e^{2\left(u_{1}+u_{2}\right)}+e^{u_{1}}\left(\kappa_{1}-z_{1}\right)+e^{u_{2}}\left(\kappa_{1}+z_{1}\right)=0 .
$$

Observe that the parametrization in terms of the scalars is unnecessary, and we could simply provide the relation in terms of the constraint on the sections, $8 X^{0}+X^{1}\left(\kappa_{1}-z_{1}\right)+$ $X^{2}\left(\kappa_{1}+z_{1}\right)=0$. This constraint allows us to define the $R$ charges $r^{I}=X^{I} /\left(X^{I} P_{I}\right)$ as

$$
r^{0}=\frac{4 z_{1} \epsilon-\kappa_{1}}{16}, \quad r^{1}=\frac{1}{4}-\epsilon, \quad r^{2}=\frac{1}{4}+\epsilon .
$$

The central charge is proportional to $C_{I J K} a^{I} r^{J} r^{K}$, that in this case becomes

$$
C_{I J K} a^{I} r^{J} r^{K}=\frac{\kappa_{1} \kappa_{2}\left(3-16 \epsilon^{2}\right)-8 \epsilon\left(\kappa_{1} z_{2}+\kappa_{2} z_{1}\right)-z_{1} z_{2}\left(1-48 \epsilon^{2}\right)}{128},
$$

which is extremized at

$$
\epsilon=\frac{\kappa_{1} z_{2}+\kappa_{2} z_{1}}{4\left(3 z_{1} z_{2}-\kappa_{1} \kappa_{2}\right)} .
$$

The overall factor in the calculation of the central charge can be obtained by expressing $G_{N}^{(3)}$ in terms of $G_{N}^{(11)}$. We start by providing the relations [48]

$$
G_{N}^{(11)}=\frac{\pi^{4}}{32 N^{3}}, \quad G_{N}^{(7)}=\frac{G_{N}^{(11)}}{V o l_{S^{4}}}=\frac{3 \pi^{2}}{16 N^{3}}
$$

The central charge can then be expressed as

$$
c_{2 d}=\frac{3 R_{A d S_{3}}}{2 G_{N}^{(3)}}=\frac{3}{2} e^{f+2 g} \frac{4 \pi^{2} \eta_{\Sigma_{1}} \eta_{\Sigma_{2}}}{G_{N}^{(7)}}=8 N^{3} \eta_{\Sigma_{1}} \eta_{\Sigma_{2}} C_{I J K} a^{I} r^{* J} r^{* K}
$$

We further need to normalize the fluxes as done in [8]. It corresponds to the relation $\kappa_{i} \rightarrow 2 \kappa_{i}$ and $z_{i} \rightarrow 2 z_{i}$. The final result is

$$
c_{2 d}=\frac{\eta_{1} \eta_{2} N^{3}\left(3 \kappa_{1}^{2} \kappa_{2}^{2}+\kappa_{1}^{2} z_{2}^{2}-8 \kappa_{1} \kappa_{2} z_{1} z_{2}+\kappa_{2}^{2} z_{1}^{2}+3 z_{1}^{2} z_{2}^{2}\right)}{4\left(\kappa_{1} \kappa_{2}-3 z_{1} z_{2}\right)}
$$

that matches the one found in [8] at large $N$. The finite $N$ contribution has been obtained in [10] and involves higher derivatives in $\mathrm{AdS}_{5}$ supergravity.

\section{Conclusions}

In this paper we discussed some aspects of supersymmetric flows from $\mathrm{AdS}_{5}$ to $\mathrm{AdS}_{3} \times \Sigma_{g}$ in gauged supergravity, dual to the compactification of $4 \mathrm{~d} \mathcal{N}=1$ SCFTs on compact Riemann surfaces with a partial topological twist. On the field theory side it has been shown that the central charge of the $2 \mathrm{~d}$ theory can be obtained in terms of the anomalies of the $4 \mathrm{~d}$ theory through the formalism of the anomaly polynomial. On the holographic dual side we have 
shown that this mechanism can be reproduced in terms of the central charge of the gauging and of a superpotential. The flow is triggered in supergravity by turning on some fluxes for the vectors. The central charge can be expressed in terms of the fluxes, the isometries and the sections. By using the constraints of the Special geometry we obtained a quadratic formula for the central charge of the $2 \mathrm{~d}$ theory in terms of the R-charges, representing the mixing coefficients of the vectors with the graviphoton. The extremization of the central charge on the field theory side is associated to an attractor mechanism for the scalars on the supergravity side. We applied this formalism to a consistent truncation of $T^{1,1}$, containing a Betti vector multiplet, dual to the baryonic symmetry. This allowed us to reproduce the fact that the baryonic symmetry does not mix with the R-charge of the $4 \mathrm{~d}$ case but it can mix once the theory is compactified to $2 \mathrm{~d}$. We have shown the existence of solutions interpolating between the $\mathrm{AdS}_{5}$ and the $\mathrm{AdS}_{3}$ vacua and we have we computed the central charge from the gravitational side, reproducing the field theory results. We provided further examples of this extremization by matching with the results obtained for other truncations, corresponding to the partially topologically twisted compactification of $\mathcal{N}=4 \mathrm{SYM}$ and of infinite families of $\mathcal{N}=1$ SCFTs describing M5 branes on compact Riemann surfaces.

Our analysis can be applied to other truncations in presence of vector multiplets and hypermultiplets. For example it may be interesting to study the $\mathcal{N}=2$ truncation of $5 \mathrm{~d}$ maximal gauged supergravity worked out in [44], corresponding to the holographic dual description of the $\mathcal{N}=1^{*}$ Leigh-Strassler fixed point [49]. By reformulating this theory in terms $5 \mathrm{~d}$ of $\mathcal{N}=2$ gauged supergravity and applying our formalism it should be possible to recover the $2 \mathrm{~d}$ central charge obtained by the twisted compactification of this model on a hyperbolic Riemann surface. Another interesting line of research consists in including the effect of higher derivatives, along the lines of [10]. This corresponds to including the Pontryagin classes in the calculation of the central charge [12], and it is related to subleading effects in the rank of the gauge groups.

Moreover, the existence of a volume formula for the $\mathcal{M}_{7}$ manifold was conjectured in [12]. Such volume formula should reproduce the central charge and its extremization similarly to the case of $\mathrm{SE}_{5}$ discussed in [50]. In this paper we obtained an expression for $c_{2 d}$ that is quadratic in the sections and is formulated in terms of the CS terms of the $5 \mathrm{~d}$ theory. The CS terms are associated to triangle anomalies and the volumes of the $\mathrm{SE}_{5}$ geometry [51]. This formulation looks a promising starting point to construct the volume formula for the $\mathcal{M}_{7}$ manifold in terms of the geometric data of the $\mathrm{SE}_{5}$ geometry.

We wish to conclude our discussion by stressing that the connection between the extremization principles and the attractor mechanism extends beyond the five-dimensional case. For example, it has been shown recently in [36] that the microstate counting for $\mathrm{AdS}_{4}$ black holes can been achieved through the extremization of a topologically twisted index on $S^{2} \times S^{1}$. This extremization principle corresponds to the $\mathrm{AdS}_{2}$ attractor. It would be interesting to extend the analysis performed here to the case of the $\mathcal{N}=2 \mathrm{AdS}_{4}$ supergravity truncations in presence of Betti multiplets, performed in [52]. Investigation of the extremization principle in such theories might shed light on the microscopic origin of the $\mathrm{AdS}_{4}$ BPS black holes found in [42] by exploiting the results found in [53, 54]. 


\section{Acknowledgments}

We are grateful to D. Cassani, L. Cassia, K. Hristov, S. Penati and P. Szepietowski for useful comments and discussions, and R. Monten for help in the analysis of the numerical solutions in section 5.3 and collaboration in related projects. We acknowledge support from the Simons Center for Geometry and Physics, Stony Brook University for hospitality during some steps of this paper. The work of A. A. is supported by the Swiss National Science Foundation (snf) under grant number pp00p2_157571/1. C. T. acknowledges support from the NWO Rubicon grant, Columbia University and from DOE grant DE-SC0011941.

\section{A $\mathcal{N}=25 \mathrm{~d}$ gauged supergravity}

In this appendix we review some basic aspects of $5 \mathrm{~d}$ Gauged supergravity and we fix the conventions we use in the main body of the paper for the analysis of the truncation of $T^{1,1}$ with the Betti vector multiplet turned on. The general matter content of $\mathcal{N}=2$ supergravity consists in the gravity multiplet, and vector, tensor and hypermultiplets. In the $T^{1,1}$ truncation taken into consideration tensor multiplets do not appear, hence we do not consider them here. We refer the reader to [55] (and [56] as well) for a more general discussion of gauged $\mathcal{N}=25 \mathrm{~d}$ supergravity. In what follows we will stick to the conventions used in [41].

The bosonic field content of the supergravity theory is as follows. The gravity multiplet consists in the metric $g_{\mu \nu}$ and one vector $A_{\mu}^{0}$. We have then $n_{v}$ vector multiplets, which consist in $n_{v}$ vectors $A_{\mu}^{I}, I=1 \ldots, n_{v}$, and vector multiplet scalars $\phi^{x}$, living on a Very Special manifold. There are finally $n_{h}$ hypermultiplets, for a total of $4 n_{h}$ hyperscalars $q^{X}$ which parameterize a Quaternionic manifold. The bosonic $\mathcal{N}=2$ Lagrangian has this form:

$$
\begin{aligned}
\mathcal{L}= & R-\frac{1}{2} g_{x y} D_{\mu} \phi^{x} D^{\mu} \phi^{y}-\frac{1}{2} g_{X Y} D_{\mu} q^{X} D^{\mu} q^{Y} \\
& -\frac{1}{4} G_{I J} F_{\mu \nu}^{I} F^{J \mu \nu}+\frac{1}{24} c_{I J K} \epsilon^{\mu \nu \rho \lambda \sigma} F_{\mu \nu}^{I} F_{\rho \lambda}^{J} A_{\sigma}^{K}-V .
\end{aligned}
$$

The scalar potential is

$$
V=2 g^{x y} \partial_{x} X^{I} \partial_{y} X^{J} P_{I}^{r} P_{J}^{r}-\frac{4}{3}\left(P_{I}^{r} X^{I}\right)^{2}+\frac{1}{2} g_{X Y} K_{I}^{X} K_{J}^{Y} X^{I} X^{J},
$$

and the covariant derivatives for the vector multiplet and for the hypermultiplet scalars (fixing the gauge coupling $g=1$ ) are defined as

$$
D_{\mu} \phi^{x}=\partial_{\mu} \phi^{x}+A_{\mu}^{I} K_{I}^{x}\left(\phi^{x}\right), \quad D_{\mu} q^{X}=\partial_{\mu} q^{X}+A_{\mu}^{I} K_{I}^{X}\left(q^{X}\right),
$$

where $K_{I}^{x}\left(\phi^{x}\right)$ and $K_{I}^{X}\left(q^{X}\right)$ are Killing vectors corresponding to the gauging of the isometries of the Special manifold and Quaternionic manifold.

The supersymmetry variations for the gravitino, gauginos and hyperinos of [55] adapted to our conventions $[41,57]$ read

$$
\begin{aligned}
\delta \psi_{\mu i} & =\left[D_{\mu}+\frac{i}{24} X_{I}\left(\gamma_{\mu}{ }^{\nu \rho}-4 \delta_{\mu}^{\nu} \gamma^{\rho}\right) F_{\nu \rho}^{I}\right] \epsilon_{i}+\frac{i}{6} \gamma_{\mu} X^{I}\left(P_{I}\right)_{i}{ }^{j} \epsilon_{j}, \\
\delta \lambda_{i}^{x} & =\left(-\frac{i}{2} \gamma \cdot D \phi^{x}-\frac{1}{4} g^{x y} \partial_{y} X_{I} \gamma^{\mu \nu} F_{\mu \nu}^{I}\right) \epsilon_{i}-g^{x y} \partial_{y} X^{I}\left(P_{I}\right)_{i}{ }^{j} \epsilon_{j}, \\
\delta \zeta^{A} & =f_{X}^{i A}\left(-\frac{i}{2} \gamma \cdot D q^{X}+\frac{1}{2} X^{I} K_{I}^{X}\right) \epsilon_{i},
\end{aligned}
$$


The supercovariant derivative acting on the supersymmetry parameter is

$$
D_{\mu} \epsilon_{i}=\partial_{\mu} \epsilon_{i}+\frac{1}{4} w_{\mu}^{a b} \gamma_{a b} \epsilon_{i}+\hat{\omega}_{\mu}^{x}\left(\sigma^{x}\right)_{i}^{j} \epsilon_{j}+\frac{1}{2} A_{\mu}^{I}\left(P_{I}\right)_{i}{ }^{j} \epsilon_{j}
$$

where $\hat{\omega}_{\mu}=\frac{i}{2} \partial_{\mu} q^{u} \omega_{u}^{x}$ is the connection on the Quaternionic manifold.

So in total the susy variation of the gravitino reads:

$$
\begin{aligned}
\delta \psi_{\mu i}= & \partial_{\mu} \epsilon_{i}+\frac{1}{4} w_{\mu}^{a b} \gamma_{a b} \epsilon_{i}+\hat{\omega}_{\mu}^{x}\left(\sigma^{x}\right)_{i}^{j} \epsilon_{j}+\frac{1}{2} A_{\mu}^{I}\left(P_{I}\right)_{i}{ }^{j} \epsilon_{j} \\
& +\frac{i}{24} X_{I}\left(\gamma_{\mu}{ }^{\nu \rho}-4 \delta_{\mu}^{\nu} \gamma^{\rho}\right) F_{\nu \rho}^{I} \epsilon_{i}+\frac{i}{6} X^{I} \gamma_{\mu}\left(P_{I}\right)_{i}{ }^{j} \epsilon_{j} .
\end{aligned}
$$

The vector multiplet scalars $\phi^{x}$ are often expressed in terms of the scalars $X^{I}\left(\phi^{x}\right)$, constrained by $\frac{1}{6} C_{I J K} X^{I} X^{J} X^{K}=1$. Moreover, the metric on the scalar manifold is given by

$$
G_{I J}=X_{I} X_{J}-C_{I J K} X^{K}, \quad g_{x y}=\partial_{x} X^{I} \partial_{y} X^{J} G_{I J},
$$

with the $X_{I}$ fields (lower index) given by

$$
X_{I}=\frac{1}{2} c_{I J K} X^{J} X^{K} .
$$

The fields $f_{i}^{A X}$ are the vielbeins on the Quaternionic manifold [55], with the SU(2) index $i=1,2$ and the $\operatorname{Sp}\left(2 n_{h}\right)$ index $A=1, \ldots, 2 n_{h}$ raised and lowered as usual by the matrices $\varepsilon^{i j}$ and $C_{A B}$. The metric on the Quaternionic manifold is

$$
g_{X Y}=f_{X}^{i A} f_{Y}^{j B} \varepsilon_{i j} C_{A B}=f_{X}^{i A} f_{Y i A} .
$$

The $\mathrm{SU}(2)$ curvature is

$$
\Omega_{X Y i j}=f_{X C(i} f_{j) Y}^{C},
$$

and the connection $\omega_{\mu, i}^{j}=\left(\partial_{\mu} q^{X}\right) \omega_{X, i}^{j}$ is such that

$$
\begin{gathered}
\Omega_{X Y i}^{j}=i \Omega_{X Y}^{r}\left(\sigma_{r}\right)_{i}^{j}=2 \partial_{[X} \omega_{Y] i}^{j}-2 \omega_{[X|i|}^{k} \omega_{Y] k}^{j}, \\
\Omega^{r}=d \omega^{r}-\varepsilon^{r s t} \omega^{s} \omega^{t} .
\end{gathered}
$$

The SU(2) curvatures are proportional to the KyperKahler forms, hence

$$
\Omega_{X Y}^{r} \Omega^{s Y Z}=-\frac{1}{4} \delta^{r s} \delta_{X}^{Z}-\frac{1}{2} \varepsilon^{r s t} \Omega_{X}^{t}{ }^{Z} .
$$

Finally, the Killing prepotentials $\left(P_{I}\right)_{i}^{j}=P_{I}^{r}\left(i \sigma^{r}\right)_{i}^{j}$ are related to the Killing vectors $K_{I}^{X}$ by the equation

$$
K_{I}^{X} \Omega_{X Y}^{r}=D_{Y} P_{I}^{r}, \quad D_{X} P_{I}^{r} \equiv \partial_{X} P_{I}^{r}+2 \varepsilon^{r s t} \omega_{X}^{s} P_{I}^{t} .
$$

It is useful to define the following quantities:

$$
P^{r} \equiv X^{I} P_{I}^{r}, \quad K^{X} \equiv X^{I} K_{I}^{X} .
$$

We moreover decompose the vector $P^{r}$ in its norm and phase

$$
P^{r}=W Q^{r}, \quad Q^{r} Q^{r}=1, \quad W=\sqrt{P^{r} P^{r}} .
$$

Lastly, one can straightforwardly verify the relation

$$
\partial_{X} W=\frac{1}{W} P^{r} D_{X} P_{r}=\frac{1}{W} P^{r} \Omega_{X Y}^{r} K^{Y}
$$

which will be useful later. 


\section{B BPS equations}

We give here a detailed derivation of the BPS equations, which are obtained by setting to zero the supersymmetry variations of the gravitino, gaugino and hyperino. In doing so, we plug the ansatz of the metric (3.1)(3.3) and purely magnetic gauge field $A_{x}$ and $A_{y}$ (while $A_{t}=0$ ) in the supersymmetry variations (A.4). We make the following ansatz for the Killing spinor projection relations, where the numbers indicate flat indices:

$$
\gamma_{2} \epsilon_{i}=-Q^{r} \sigma^{r}{ }_{i j} \epsilon^{j}, \quad \gamma_{34} \epsilon_{i}=-i Q^{r} \sigma^{r}{ }_{i j} \epsilon^{j}, \quad \partial_{t} \epsilon=\partial_{z} \epsilon=\partial_{\mathrm{x}} \epsilon=\partial_{\mathrm{y}} \epsilon=0 .
$$

Imposing these projectors amount to preserving $1 / 4$ of the supersymmetries throughout the flow. For the solutions taken into consideration, there is a supersymmetry enhancement at the IR: the $A d S_{3} \times \Sigma_{g}$ geometry is in fact $1 / 2$ BPS - see discussion in section 5.3.

We will make the following choice of vielbeins

$$
e_{t}^{0}=e_{z}^{1}=e_{r}^{2}=e^{f(r)}, \quad e_{\mathrm{x}}^{3}=e^{g(r)} \quad e_{\mathrm{y}}^{4}=e^{g(r)} F(\mathrm{x}),
$$

where the function $F$ is defined in (3.3). The non vanishing components of the spin connection are:

$$
w_{t}^{02}=w_{z}^{12}=f^{\prime}, \quad w_{\mathrm{x}}^{23}=-g^{\prime} e^{g-f}, \quad w_{\mathrm{y}}^{24}=-g^{\prime} e^{g-f} F(\mathrm{x}), \quad w_{\mathrm{y}}^{34}=-F^{\prime}(\mathrm{x}) .
$$

The analysis which follows apply to the case $k= \pm 1$, but the procedure can be straightforwardly applied to the $\kappa=0$ case as well.

\section{B.1 Gravitino}

The BPS equation derived from the $t$ component of the gravitino variation $\delta \psi_{t i}$ reads

$$
\partial_{t} \epsilon_{i}+\frac{1}{4} w_{t}^{02} \gamma_{02} \epsilon_{i}+\frac{i}{24} X_{I} \gamma_{t}^{\mathrm{xy}} F_{\mathrm{xy}}^{I} \epsilon_{i}+\frac{i}{6} \gamma_{t} X^{I} P_{I_{i}^{j}}^{j} \epsilon_{j}=0
$$

We impose now the relations (B.1). Therefore, the first term drops out, and the other give the equation

$$
-\frac{1}{2} f^{\prime} \gamma_{0} Q^{r} \sigma^{r}{ }_{i j} \epsilon^{j}-\frac{X_{I} a^{I}}{12} e^{-2 g+f} \gamma_{0} Q^{r} \sigma^{r}{ }_{i j} \epsilon^{j}-\frac{1}{6} \gamma_{0} e^{f} P^{r} \sigma^{r}{ }_{i j} \epsilon^{j}=0,
$$

which, given (A.17), can be simplified in

$$
f^{\prime}+\frac{1}{6} a^{I} X_{I} e^{-2 g+f}+\frac{1}{3} e^{f} W=0 .
$$

From the $r$-component, using (B.1) we have

$$
\partial_{r} \epsilon_{i}+\hat{\omega}_{\mu}^{x}\left(\sigma^{x}\right)_{i}^{j} \epsilon_{j}-\frac{i}{24} a^{I} X_{I} e^{-2 g+f} \gamma_{2}^{34} \epsilon_{i}+\frac{i}{6} \gamma_{2} e^{f} X^{I} P_{I_{i}}^{j} \epsilon_{j}=0 .
$$

which determines the functional form of in terms of $r$ of the Killing spinor $\epsilon_{i}(r)$.

The $\mathrm{x}$ component of the gravitino variation gives:

$$
\partial_{\mathrm{x}} \epsilon_{i}+\frac{1}{4} w_{\mathrm{x}}^{23} \gamma_{23} \epsilon_{i}-\frac{i}{6} \gamma_{\mathrm{y}} a^{I} X_{I} \epsilon_{i}+\frac{i}{6} \gamma_{\mathrm{x}} X^{I} P_{I_{i}}^{j} \epsilon_{j}=0,
$$


which after using (B.1) yields

$$
g^{\prime}-\frac{1}{3} X_{I} a^{I} e^{-2 g+f}+\frac{1}{3} e^{f} W=0 .
$$

The y component of the gravitino variation gives:

$$
\partial_{\mathrm{y}} \epsilon_{i}+\frac{1}{4} w_{\mathrm{y}}^{23} \gamma_{23} \epsilon_{i}+\frac{1}{4} w_{\mathrm{y}}^{34} \gamma_{34} \epsilon_{i}+\frac{1}{2} A_{\mathrm{y}}^{I}\left(P_{I}\right)_{i}^{j} \epsilon_{j}-\frac{i}{6} \gamma_{\mathrm{x}} a^{I} X_{I} \epsilon_{i}+\frac{i}{6} \gamma_{\mathrm{y}} X^{I} P_{I_{i}}^{j} \epsilon_{j}=0 .
$$

Imposing the projection relations (B.1), we obtain two parts, one multiplying $Q^{r} \sigma^{r}{ }_{i j}$ and the other $\gamma_{\mathrm{y}} Q^{r} \sigma^{r}{ }_{i j}$, that need to vanish separately. From the terms in $\gamma_{\mathrm{y}} Q^{r} \sigma^{r}{ }_{i j}$ we get exactly equation (B.8) obtained before. The part multiplying $P_{I_{i}^{j}}^{j} \epsilon_{j}$ yields

$$
\frac{1}{2} A_{\mathrm{y}}^{I} P_{I}^{r}=\frac{\kappa}{2} Q^{r} F^{\prime}(\mathrm{x}) \quad \rightarrow \quad a^{I} P_{I}^{r}=-\kappa Q^{r},
$$

where we made use of the relations (5.8).

Finally, the $z$ component of the gravitino variation gives exactly the same equation (B.6).

\section{B.2 Gaugino}

After plugging in the ansatz (3.1)(3.3)(3.4), and retaining only the radial dependence of the fields, the gaugino equation reads

$$
-\frac{i}{2} \gamma^{r} \partial_{r} \phi^{x} \epsilon_{i}-\frac{i}{2} \gamma^{\phi} a^{I} K_{I}^{x} \epsilon_{i}-\frac{i}{2} \gamma^{\theta} a^{I} K_{I}^{x} \epsilon_{i}+\frac{1}{2} g^{x y} \partial_{y} X_{I} \gamma^{\mathrm{xy}} a^{I} \epsilon_{i}-\frac{1}{2} g^{x y} \partial_{y} X^{I}\left(P_{I}\right)_{i}^{j} \epsilon_{j}=0 .
$$

Since the models taken into consideration don't gauge the isometries of the Special manifold, we have $K_{I}^{x}=0$. Hence, after using the projections (B.1) we are left with the equations

$$
\frac{1}{2} \partial_{r} \phi^{x} Q^{r}+\frac{1}{2} e^{-2 g+f} g^{x y} \partial_{y} X_{I} a^{I} Q^{r}-e^{f} g^{x y} \partial_{y} X^{I} P_{I}^{r}=0 .
$$

\section{B.3 Hyperino}

The hyperino equation instead requires a bit more effort. Plugging in all components, and taking into account the radial dependence of the scalar fields, it reads

$$
f_{X}^{i A}\left(-\frac{i}{2} \gamma^{r} \partial_{r} q^{X}+\gamma^{\mathrm{y}} A_{\mathrm{y}}^{I} K_{I}^{X}+\frac{1}{2} X^{I} K_{I}^{X}\right) \epsilon_{i}=0 .
$$

The second and third term should vanish separately, hence the constraint

$$
a^{I} K_{I}^{X}=0 .
$$

We are now ready to massage the remaining equation

$$
f_{X}^{i A}\left(-i \gamma^{r} \partial_{r} q^{X}+X^{I} K_{I}^{X}\right) \epsilon_{i}=0,
$$

following the procedure spelled out in [58]. We multiply the left hand side of this equations by $f_{A j}^{Y}$. Using the relations (A.10) and (A.11) we get

$$
f_{X j A} f_{Y}^{i A}=\frac{1}{2} g_{X Y} \delta_{i}^{j}+\Omega_{X Y j}{ }^{i}
$$


so that eq. (B.14), using the projection relations (B.1), becomes of the form

$$
\left[g_{X Y} \delta_{j}^{i}+2 i \Omega_{X Y}{ }^{r} \sigma_{j}^{r}{ }_{j}^{i}\right]\left[i q^{X^{\prime}} Q^{s} \sigma_{i}^{s}{ }^{k}+K^{X} \delta_{i}^{k}\right]=0,
$$

also written as a matrix equation

$$
\left[A_{Y} \delta_{j}^{k}+i B_{Y}^{r}\left(\sigma^{r}\right)_{j}^{k}\right] \epsilon_{k}=0
$$

where

$$
\begin{aligned}
& A_{Y}=K_{Y}-2 \Omega_{Y X}^{r} Q^{r} q^{X^{\prime}}, \\
& B_{Y}^{r}=g_{Y X} q^{X^{\prime}} Q^{r}+\varepsilon^{r s t} \Omega_{Y X}^{s} Q^{t} q^{X^{\prime}}+2 \Omega_{Y X}^{r} K^{X} .
\end{aligned}
$$

Both $A_{Y}$ and $B_{Y}^{r}$ need to vanish, and they do if the following condition is enforced (more details in [58])

$$
q^{Y^{\prime}} g_{Y X}=2 Q^{r} \Omega^{r} X Y K^{Y}
$$

where $W$ is defined in (A.17) and we have used (A.18). This is the last BPS equation.

To sum up, we have massaged the BPS equations in the neat system of boson equations

$$
\begin{aligned}
f^{\prime}+\frac{1}{3} e^{f} W+\frac{1}{6} a^{I} X_{I} e^{-2 g+f} & =0 \\
g^{\prime}+\frac{1}{3} e^{f} W-\frac{1}{3} a^{I} X_{I} e^{-2 g+f} & =0 \\
\frac{1}{2} \partial_{r} \phi^{x} Q^{r}+\frac{1}{2} e^{-2 g+f} g^{x y} \partial_{y} X_{I} a^{I} Q^{r}-e^{f} g^{x y} \partial_{y} X^{I} P_{I}^{r} & =0 \\
q^{Y^{\prime}} g_{Y X}-2 Q^{r} \Omega^{r} X Y K^{Y} & =0 \\
a^{I} P_{I}^{r}+\kappa Q^{r} & =0 \\
a^{I} K_{I}^{X} & =0 .
\end{aligned}
$$

Given these equations, the Maxwell's and Bianchi equations are automatically satisfied for the choice of warp factors and gauge fields done in section 5 .

Open Access. This article is distributed under the terms of the Creative Commons Attribution License (CC-BY 4.0), which permits any use, distribution and reproduction in any medium, provided the original author(s) and source are credited.

\section{References}

[1] E. Witten, Topological $\sigma$-models, Commun. Math. Phys. 118 (1988) 411 [InSPIRE].

[2] M. Bershadsky, A. Johansen, V. Sadov and C. Vafa, Topological reduction of $4 D S Y M$ to $2 D$ $\sigma$-models, Nucl. Phys. B 448 (1995) 166 [hep-th/9501096] [INSPIRE].

[3] M. Bershadsky, C. Vafa and V. Sadov, D-branes and topological field theories, Nucl. Phys. B 463 (1996) 420 [hep-th/9511222] [INSPIRE]. 
[4] J.M. Maldacena and C. Núñez, Supergravity description of field theories on curved manifolds and a no go theorem, Int. J. Mod. Phys. A 16 (2001) 822 [hep-th/0007018] [inSPIRE].

[5] A. Johansen, Holomorphic currents and duality in $N=1$ supersymmetric theories, JHEP 12 (2003) 032 [hep-th/0309125] [INSPIRE].

[6] A. Kapustin, Holomorphic reduction of $N=2$ gauge theories, Wilson-'t Hooft operators and S-duality, hep-th/0612119 [INSPIRE].

[7] A. Donos, J.P. Gauntlett and N. Kim, AdS solutions through transgression, JHEP 09 (2008) 021 [arXiv:0807.4375] [INSPIRE].

[8] F. Benini and N. Bobev, Two-dimensional SCFTs from wrapped branes and c-extremization, JHEP 06 (2013) 005 [arXiv: 1302.4451] [INSPIRE].

[9] A. Donos and J.P. Gauntlett, Flowing from $A d S_{5}$ to $A d S_{3}$ with $T^{1,1}$, JHEP 08 (2014) 006 [arXiv: 1404.7133] [INSPIRE].

[10] M. Baggio, N. Halmagyi, D.R. Mayerson, D. Robbins and B. Wecht, Higher derivative corrections and central charges from wrapped M5-branes, JHEP 12 (2014) 042 [arXiv: 1408.2538] [INSPIRE].

[11] Y. Bea et al., Compactifications of the Klebanov-Witten CFT and new AdS $S_{3}$ backgrounds, JHEP 05 (2015) 062 [arXiv: 1503.07527] [INSPIRE].

[12] F. Benini, N. Bobev and P.M. Crichigno, Two-dimensional SCFTs from D3-branes, JHEP 07 (2016) 020 [arXiv:1511.09462] [INSPIRE].

[13] S. Schafer-Nameki and T. Weigand, F-theory and 2D (0,2) theories, JHEP 05 (2016) 059 [arXiv: 1601.02015] [INSPIRE].

[14] F. Apruzzi, F. Hassler, J.J. Heckman and I.V. Melnikov, UV completions for non-critical strings, JHEP 07 (2016) 045 [arXiv: 1602.04221] [INSPIRE].

[15] F. Apruzzi, F. Hassler, J.J. Heckman and I.V. Melnikov, From 6D SCFTs to dynamic GLSMs, arXiv: 1610.00718 [INSPIRE].

[16] N. Kim, AdS $S_{3}$ solutions of IIB supergravity from D3-branes, JHEP 01 (2006) 094 [hep-th/0511029] [INSPIRE].

[17] P. Figueras, O.A.P. Mac Conamhna and E. O Colgain, Global geometry of the supersymmetric Ad $S_{3} / C F T_{2}$ correspondence in M-theory, Phys. Rev. D 76 (2007) 046007 [hep-th/0703275] [INSPIRE].

[18] S. Benvenuti, S. Franco, A. Hanany, D. Martelli and J. Sparks, An infinite family of superconformal quiver gauge theories with Sasaki-Einstein duals, JHEP 06 (2005) 064 [hep-th/0411264] [INSPIRE].

[19] J.P. Gauntlett, D. Martelli, J. Sparks and D. Waldram, Sasaki-Einstein metrics on $S^{2} \times S^{3}$, Adv. Theor. Math. Phys. 8 (2004) 711 [hep-th/0403002] [INSPIRE].

[20] K.A. Intriligator and B. Wecht, The exact superconformal $R$ symmetry maximizes a, Nucl. Phys. B 667 (2003) 183 [hep-th/0304128] [InSPIRE].

[21] M. Bertolini, F. Bigazzi and A.L. Cotrone, New checks and subtleties for AdS/CFT and a-maximization, JHEP 12 (2004) 024 [hep-th/0411249] [INSPIRE].

[22] A. Butti and A. Zaffaroni, R-charges from toric diagrams and the equivalence of a-maximization and Z-minimization, JHEP 11 (2005) 019 [hep-th/0506232] [INSPIRE]. 
[23] J. Schmude and O. Vasilakis, Superconformal symmetry in the Kaluza-Klein spectrum of warped $A d S_{3}, J H E P 10$ (2016) 096 [arXiv: 1605.00636] [INSPIRE].

[24] I.R. Klebanov and E. Witten, Superconformal field theory on three-branes at a Calabi-Yau singularity, Nucl. Phys. B 536 (1998) 199 [hep-th/9807080] [INSPIRE].

[25] D. Cassani and A.F. Faedo, A supersymmetric consistent truncation for conifold solutions, Nucl. Phys. B 843 (2011) 455 [arXiv: 1008.0883] [INSPIRE].

[26] I. Bena, G. Giecold, M. Graña, N. Halmagyi and F. Orsi, Supersymmetric consistent truncations of IIB on $T^{1,1}$, JHEP 04 (2011) 021 [arXiv: 1008.0983] [INSPIRE].

[27] P. Karndumri and E. O Colgain, Supergravity dual of c-extremization, Phys. Rev. D 87 (2013) 101902 [arXiv:1302.6532] [INSPIRE].

[28] I. Bah, C. Beem, N. Bobev and B. Wecht, Four-dimensional SCFTs from M5-branes, JHEP 06 (2012) 005 [arXiv: 1203.0303] [INSPIRE].

[29] D. Klemm, N. Petri and M. Rabbiosi, Black string first order flow in $N=2, D=5$ abelian gauged supergravity, JHEP 01 (2017) 106 [arXiv:1610.07367] [INSPIRE].

[30] Y. Tachikawa, Five-dimensional supergravity dual of a-maximization, Nucl. Phys. B 733 (2006) 188 [hep-th/0507057] [INSPIRE].

[31] E. Barnes, E. Gorbatov, K.A. Intriligator and J. Wright, Current correlators and AdS/CFT geometry, Nucl. Phys. B 732 (2006) 89 [hep-th/0507146] [INSPIRE].

[32] J.D. Brown and M. Henneaux, Central charges in the canonical realization of asymptotic symmetries: an example from three-dimensional gravity, Commun. Math. Phys. 104 (1986) 207 [INSPIRE].

[33] B. de Wit, I. Herger and H. Samtleben, Gauged locally supersymmetric D $=3$ nonlinear o-models, Nucl. Phys. B 671 (2003) 175 [hep-th/0307006] [INSPIRE].

[34] P. Karndumri and E.O. Colgáin, 3D supergravity from wrapped D3-branes, JHEP 10 (2013) 094 [arXiv: 1307.2086] [INSPIRE].

[35] A. Amariti and A. Gnecchi, $3 D \tau_{R R}$-minimization in AdS $S_{4}$ gauged supergravity, JHEP 07 (2016) 006 [arXiv : 1511.08214] [INSPIRE].

[36] F. Benini, K. Hristov and A. Zaffaroni, Black hole microstates in AdS $S_{4}$ from supersymmetric localization, JHEP 05 (2016) 054 [arXiv:1511.04085] [INSPIRE].

[37] F. Benini and A. Zaffaroni, A topologically twisted index for three-dimensional supersymmetric theories, JHEP 07 (2015) 127 [arXiv:1504.03698] [INSPIRE].

[38] A. Buchel and J.T. Liu, Gauged supergravity from type IIB string theory on $Y^{p, q}$ manifolds, Nucl. Phys. B 771 (2007) 93 [hep-th/0608002] [INSPIRE].

[39] J. Louis, P. Smyth and H. Triendl, Supersymmetric vacua in $N=2$ supergravity, JHEP 08 (2012) 039 [arXiv: 1204.3893] [INSPIRE].

[40] A. Ceresole, G. Dall'Agata and R. D'Auria, $K K$ spectroscopy of type IIB supergravity on $A d S_{5} \times T^{1} 1$, JHEP 11 (1999) 009 [hep-th/9907216] [INSPIRE]

[41] N. Halmagyi, J.T. Liu and P. Szepietowski, On $N=2$ truncations of IIB on $T^{1,1}$, JHEP 07 (2012) 098 [arXiv: 1111.6567] [INSPIRE].

[42] N. Halmagyi, M. Petrini and A. Zaffaroni, BPS black holes in AdS 4 from M-theory, JHEP 08 (2013) 124 [arXiv: 1305.0730] [INSPIRE]. 
[43] C.P. Herzog, I.R. Klebanov, S.S. Pufu and T. Tesileanu, Emergent quantum near-criticality from baryonic black branes, JHEP 03 (2010) 093 [arXiv:0911.0400] [INSPIRE].

[44] N. Bobev, K. Pilch and O. Vasilakis, (0,2) SCFTs from the Leigh-Strassler fixed point, JHEP 06 (2014) 094 [arXiv: 1403.7131] [inSPIRE].

[45] P. Karndumri and E. O. Colgáin, 3D supergravity from wrapped M5-branes, JHEP 03 (2016) 188 [arXiv: 1508.00963] [INSPIRE].

[46] P. Szepietowski, Comments on a-maximization from gauged supergravity, JHEP 12 (2012) 018 [arXiv: 1209.3025] [INSPIRE].

[47] D. Gaiotto, $N=2$ dualities, JHEP 08 (2012) 034 [arXiv:0904.2715] [INSPIRE].

[48] M. Henningson and K. Skenderis, The holographic Weyl anomaly, JHEP 07 (1998) 023 [hep-th/9806087] [INSPIRE].

[49] R.G. Leigh and M.J. Strassler, Exactly marginal operators and duality in four-dimensional $N=1$ supersymmetric gauge theory, Nucl. Phys. B 447 (1995) 95 [hep-th/9503121] [INSPIRE].

[50] D. Martelli, J. Sparks and S.-T. Yau, The geometric dual of a-maximisation for toric Sasaki-Einstein manifolds, Commun. Math. Phys. 268 (2006) 39 [hep-th/0503183] [INSPIRE].

[51] S. Benvenuti, L.A. Pando Zayas and Y. Tachikawa, Triangle anomalies from Einstein manifolds, Adv. Theor. Math. Phys. 10 (2006) 395 [hep-th/0601054] [InSPIRE].

[52] D. Cassani, P. Koerber and O. Varela, All homogeneous $N=2 M$-theory truncations with supersymmetric $A d S_{4}$ vacua, JHEP 11 (2012) 173 [arXiv:1208.1262] [INSPIRE].

[53] S.M. Hosseini and A. Zaffaroni, Large- $N$ matrix models for $3 d \mathcal{N}=2$ theories: twisted index, free energy and black holes, JHEP 08 (2016) 064 [arXiv: 1604.03122] [INSPIRE].

[54] S.M. Hosseini and N. Mekareeya, Large- $N$ topologically twisted index: necklace quivers, dualities and Sasaki-Einstein spaces, JHEP 08 (2016) 089 [arXiv:1604.03397] [INSPIRE].

[55] A. Ceresole and G. Dall'Agata, General matter coupled $N=2, D=5$ gauged supergravity, Nucl. Phys. B 585 (2000) 143 [hep-th/0004111] [INSPIRE].

[56] E. Bergshoeff, S. Cucu, T. de Wit, J. Gheerardyn, S. Vandoren and A. Van Proeyen, $N=2$ supergravity in five-dimensions revisited, Class. Quant. Grav. 21 (2004) 3015 [hep-th/0403045] [INSPIRE].

[57] J.T. Liu and P. Szepietowski, Supersymmetry of consistent massive truncations of IIB supergravity, Phys. Rev. D 85 (2012) 126010 [arXiv:1103.0029] [InSPIRE].

[58] A. Ceresole, G. Dall'Agata, R. Kallosh and A. Van Proeyen, Hypermultiplets, domain walls and supersymmetric attractors, Phys. Rev. D 64 (2001) 104006 [hep-th/0104056] [INSPIRE]. 